\title{
RMS envelope matching of electron beams from "zero" current to extreme space charge in a fixed lattice of short magnets
}

\author{
S. Bernal, H. Li, ${ }^{*}$ R. A. Kishek, B. Quinn, M. Walter, M. Reiser, ${ }^{\dagger}$ and P. G. O’Shea ${ }^{\dagger}$ \\ Institute For Research in Electronics and Applied Physics, University of Maryland, College Park, Maryland 20742, USA \\ C. K. Allen \\ Los Alamos National Laboratory, Los Alamos, New Mexico 87544, USA
}

(Received 3 June 2005; revised manuscript received 13 April 2006; published 29 June 2006)

\begin{abstract}
We present detailed calculations of RMS-envelope matching over a broad range of beam intensities for the University of Maryland Electron Ring (UMER). Containment of beams from zero current to extreme space charge, all without changing the strength of external focusing in the periodic lattice, is possible thanks to the high density of quadrupoles in UMER. In turn, the small-aspect ratio of the UMER magnets results in gradient or field profiles that are "all edges," thus requiring special treatment when constructing accurate hard-edge models. Further, the results of matching calculations, for both symmetric and asymmetric FODO (alternating gradient) schemes, are compared with calculations from simple general expressions valid in the uniform-focusing approximation of the periodic lattice. Finally, some aspects of the source-to-FODO matching calculation/optimization problem are discussed, together with sensitivity studies of the matching solutions under realistic conditions. The examples from the UMER project, which include experimental results, emphasize the practical aspects of beam envelope matching.
\end{abstract}

DOI: 10.1103/PhysRevSTAB.9.064202

PACS numbers: 29.27.Eg, 41.85.Ja

\section{INTRODUCTION}

Envelope matching, or betatron-function matching, of a beam into a periodic focusing lattice is a problem that must be solved early in the development of many particle accelerators. The matched beam "state" corresponds to a steady macroscopic situation whereby the effective beam sizes in the two transverse directions vary periodically and with constant average values along the periodic focusing lattice. The situation is similar to a tuned laser cavity, where the optics of the back-and-forth reflections of the radiation between cavity mirrors is equivalent to an infinite periodic focusing system. In the case of beams, matching may or may not be accompanied by a true "equilibrium" state in a thermal sense. However, since this is an issue outside the scope of the paper, it is sufficient to say that a matched beam is a desired condition that must be met as close as possible in order to minimize particle losses, emittance growth, and halo formation [1,2]. A different kind of matching, dispersion matching, which relates to the spread in linear momentum in the beam particles, will not be discussed here.

A general discussion of betatron (and dispersion) matching with variable-geometry schemes can be found in [3]; the treatment is based on thin lenses and zero space charge and provides analytical results for quadrupole doublets, triplets, and others. A brief discussion of betatron (and dispersion) function matching specially applicable to collider storage rings appears in $[4,5]$. Special lattice geome-

\footnotetext{
*Present address: Microsoft Corp., Redmond, WA 98052.

${ }^{\dagger}$ Also at Department of Electrical and Computer Engineering, University of Maryland, College Park, MD.
}

tries such as low-beta and Collins insertions are described in the latter references. A more general account of beam transport including space charge, lens modeling, and envelope matching appears in a recent paper by Lund and Bukh [6]. Another review of beam envelope simulation can be found in a Los Alamos unpublished report [7]. Furthermore, specific examples of envelope matching are described in a number of conference papers (e.g., $[8,9])$ and numerous internal reports from labs and institutions around the world. In this paper, we bring together all key aspects of envelope matching within a linear model that includes space charge and apply them to the University of Maryland Electron Ring (UMER) [10].

We present results of RMS envelope matching of spacecharge dominated as well as emittance-dominated beams, using the same matching geometry and without changing the strength of external focusing in the periodic lattice. We show that containment of beams over such a broad range of intensities is possible thanks to the high density of quadrupoles in UMER, a feature not to be found in any other circular machine. (Previous work, published by some of us, related to matching of a space-charge dominated electron beam in a prototype experiment [11].) Further, we revisit the basic characterization of the short UMER magnets and show how proper modeling is important for envelope matching in special cases. The calculations and experiments presented relate in most cases to UMER, but the methods used to characterize the magnets and to approach RMS envelope matching design and calculations have general applicability.

We review in the next section the RMS envelope equations which are the basis for most matching calculations. 
Models for the gradient profile of the short UMER quadrupole and for the field profile of a short solenoid are presented in Sec. III. In Sec. IV, we discuss the envelope solution in a unit FODO [Focusing(F)-Drift(O)Defocusing(D)-Drift(O)] cell, for both symmetric and asymmetric focusing. Section V contains general considerations for designing a matching section, including specific examples from UMER. In Sec. VI, we investigate the sensitivity of the matching solutions via Monte Carlo calculations. The last section is devoted to summary and conclusions. Finally, the specifics of the envelope code SPOT are presented in an appendix.

\section{MODEL BEAM AND RMS ENVELOPE EQUATIONS}

The charged-particle beam implicit throughout the paper is continuous and perfectly centered in the transport pipe. Furthermore, no change in beam energy, either intrinsic or from acceleration stages, is assumed. The beam is brought to a "matched" state in a periodic focusing lattice, after traversing a relatively short matching/injection section. The beam particle distribution undergoes an evolution from the source through the matching section, but we are not concerned with the details of such evolution. For any beam distribution having elliptical symmetry, an equivalent Kapchinskij-Vladimirskij (or K-V) distribution $[12,13]$ can be defined, and (coupled) envelope equations derived for the effective 2RMS beam dimensions in the transverse directions, $X(s)$ and $Y(s)[1]$ :

$$
\begin{aligned}
& X^{\prime \prime}(s)+\kappa_{x}(s) X(s)-\frac{2 K}{X(s)+Y(s)}-\frac{\varepsilon_{x}^{2}}{X^{3}(s)}=0, \\
& Y^{\prime \prime}(s)+\kappa_{y}(s) Y(s)-\frac{2 K}{X(s)+Y(s)}-\frac{\varepsilon_{y}^{2}}{Y^{3}(s)}=0,
\end{aligned}
$$

where primes denote derivatives with respect to the axial coordinate $s$. Further, $K=2\left(I / I_{0}\right)\left(1 / \beta^{3} \gamma^{3}\right)$ is the generalized beam perveance, $I_{0}=17 \mathrm{kA}$, approximately, for electrons, $\beta=v / c, \gamma=\left(1-\beta^{2}\right)^{-1 / 2}$ ( $c$ is the speed of light, and $v$ is the particle's velocity), and $\epsilon_{x, y}$ are the 4RMS unnormalized emittances, sometimes called "edge" emittances. The latter quantities are assumed constant in all calculations. The lattice focusing functions $\kappa_{x, y}(s)$ in Eq. (1) are explicitly defined below.

Two important parameters that characterize the solutions of Eqs. (1) with and without space charge are the phase advances of single-particle motion in one lattice period, or $\sigma$ and $\sigma_{0}$ :

$$
\sigma_{x}=\varepsilon_{x} \int_{\text {period }} \frac{d s}{X^{2}(s)}, \quad \sigma_{0 x}=\varepsilon_{x} \int_{\text {period }} \frac{d s}{X_{0}^{2}(s)},
$$

and similarly for the $y$-plane. Above, $X_{0}$ is the envelope solution without space charge. The ratios $\sigma_{x, y} / \sigma_{0 x, y}$ define the tune depressions in the $x, y$-planes, key parameters in the description of space-charge dominated beam transport $[1,2]$.

The envelope equations, Eqs. (1), are based on the motion of paraxial particles under the action of linear fields from external focusing and the linear self-fields of the equivalent $\mathrm{K}-\mathrm{V}$ distribution. We emphasize that the $\mathrm{K}-\mathrm{V}$ distribution is just a useful mathematical construct and that no amount of beam manipulation at or near the source will result in a close realization of such an ideal distribution. Collimating apertures can certainly yield a nearly uniform particle-density distribution, but simple, similar control of the velocity flow profile is not possible. Even if the latter were possible to a high degree, thermal effects would always remain. Thus, solutions of the envelope equations that disregard the emittance term are necessarily unrealistic, even in the limit of extreme space-charge dominated transport.

A simplification useful for zero-order design calculations is provided by the uniform-focusing, or smooth approximation [1], which we will employ in Sec. IV. The approximation can be fully justified mathematically and its applicability studied for a few cases of idealized focusing functions [14]. Further, precise solutions of the envelope equations have been developed employing special power expansions [15].

\section{UMER MAGNETS}

Figure 1 shows the schematics of the matching/injection section in UMER employed for DC injection experiments, i.e., for beam transport over one turn only. The section consists of a short solenoid and six printed-circuit (PC) quadrupoles distributed over a length of $1.4 \mathrm{~m}$, approximately. Although the PC quadrupoles have been extensively characterized [16], we present additional calculations in this section, together with a new treatment of the short solenoid. The methods are especially relevant to small-aspect ratio elements, but show the importance of properly modeling of lenses for matching calculations.

\section{A. Printed-circuit quadrupoles}

The on-axis gradient profile of the short, air-core magnetic UMER quadrupoles is shown in Fig. 2. It is obtained

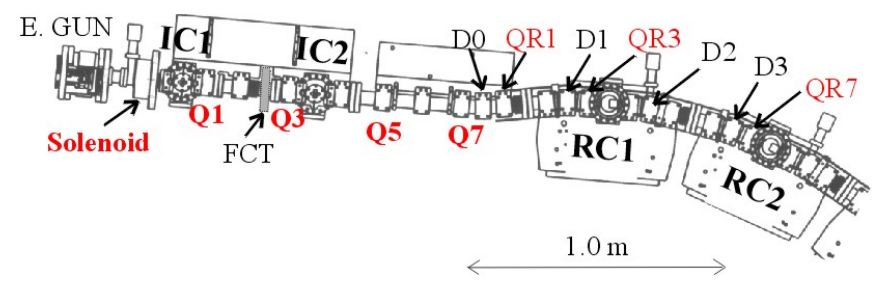

FIG. 1. (Color) Matching/injection section and first ring diagnostics chambers (RC) in UMER. All focusing/bending elements, including the injection dipole (D0) and quadrupoles, were DC operated at this stage of UMER. 

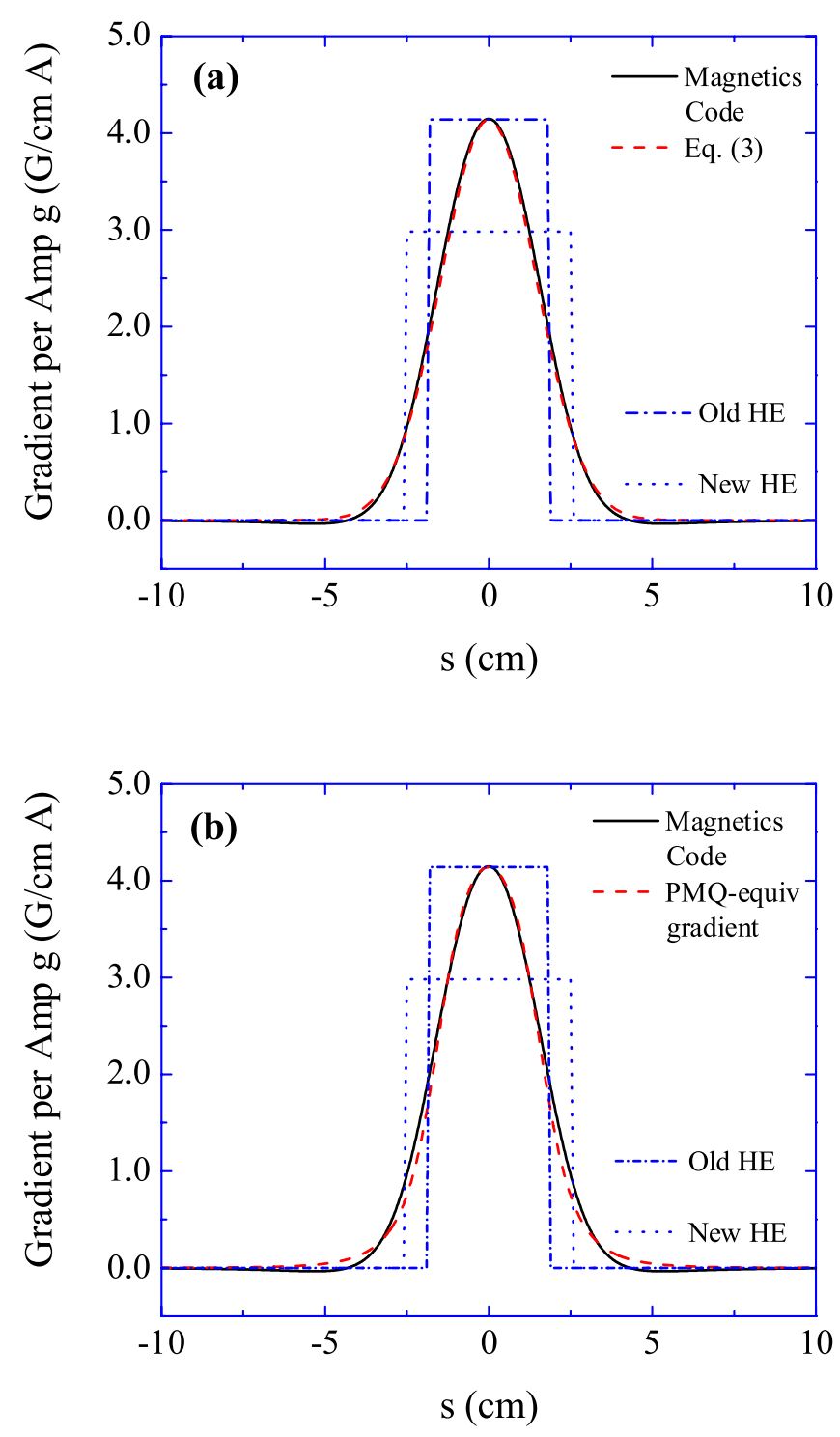

FIG. 2. (Color) On-axis gradient (per Amp) profile of UMER air-core, printed-circuit quadrupole (2nd generation): (a) calculated, analytical, and hard-edge (HE) profiles, and (b) calculated, PMQ-equivalent gradient, and hard-edge (HE) profiles.

with the help of a magnetics code developed by one of us (Li). The profile can be approximated with the following function [17]:

$$
g(s)=g_{0} \exp \left(-s^{2} / d^{2}\right),
$$

where $g_{0}=4.14 \mathrm{G} / \mathrm{cm} \mathrm{A}$ is the on-axis peak gradient per amp, and $d=2.05 \mathrm{~cm}$. The function is also shown in Fig. 2(a).

Traditionally, the effective length of a magnet is calculated from the equation

$$
l=\frac{1}{g_{0}} \int_{-\infty}^{\infty} g(s) d s
$$

where $g(s)$ is the on-axis field gradient, and $g_{0}$ is the peak value. The field gradient is related to $\kappa_{x, y}(s)$ of Eqs. (1) by

$$
\kappa_{x}(s)=\frac{q}{p_{0}} g(s), \quad \kappa_{y}(s)=\frac{-q}{p_{0}} g(s),
$$

where $q$ and $p_{0}$ are the particle's charge and (design) linear momentum, respectively. For electrons, a positive $g(s)$ yields a defocusing effect on the horizontal, $x$-plane.

As an alternative to Eq. (3), it is possible to model the smooth-gradient profile using a fringe field function that, although originally derived for permanent magnet quadrupoles (PMQ) [18], can be adapted to any focusing element. The function is defined by

$$
\begin{aligned}
F(s)= & \frac{1}{2}\left\{1-\frac{z}{8}\left(\frac{1}{r_{1}}+\frac{1}{r_{2}}\right)\right. \\
& \left.\times\left[\frac{f_{1}^{2} f_{2}^{2}\left(f_{1}^{2}+f_{1} f_{2}+f_{2}^{2}+4+8\left(f_{1} f_{2}\right)^{-1}\right)}{f_{1}+f_{2}}\right]\right\},
\end{aligned}
$$

where

$$
f_{1,2}(s)=\left[1+\left(\frac{s}{r_{1,2}}\right)^{2}\right]^{-1 / 2},
$$

and $r_{1}, r_{2}$ are the inner and outer radius, respectively, of the real or hypothetical PMQ. The complete profile is constructed by adding two functions $F(s)$ with an overlap distance $l_{p}$ :

$$
P(s)=F\left(s-\frac{l_{p}}{2}\right)-F\left(s+\frac{l_{p}}{2}\right),
$$

so the final PMQ-equivalent gradient profile is given by

$$
g_{p m q}(s)=g_{0} \frac{P(s)}{P(0)} .
$$

A good analytical fit to any measured or calculated gradient profile can be obtained by adjusting $r_{1}, r_{2}$, and $l_{p}$. In this way, PMQ elements can be defined, for example, in the popular code TRACE 3D $[19,20]$ by specifying $r_{1}, r_{2}$ and an effective length (not $l_{p}$ ). In addition, a "fringe field extension factor" is used in TRACE (default $=2.5 r_{1}$ ) to put a limit to calculations on the gradient wings. For the second-generation air-core UMER quadrupoles, we have $r_{1}=13 \mathrm{~mm}, r_{2}=47 \mathrm{~mm}, l_{p}=3.062 \mathrm{~cm}$, and a field extension factor $10 r_{1}=13 \mathrm{~cm}$. With these values, the profiles $g(s)$ [Eq. (3)] and $g_{p m q}$ [Eq. (9)] have the same effective length, $l$, as defined in Eq. (4). Figure 2(b) shows the PMQ-equivalent gradient profile together with the profile from the magnetics code.

Although smooth-gradient profiles are more realistic, it is normal practice to model the quadrupoles as hard-edge elements, i.e., as constant focusing/defocusing lenses with gradient $\pm g_{0}$ and length $l$. These choices are in most cases acceptable when the effect of the gradient wings is small, as with long quadrupoles having a recognizable flat top gradient (e.g., the electrostatic quadrupole described in 
[6]). Although the model can be applied even to short ("all edges") quadrupoles like UMER's (see Fig. 2), the shortcomings of the model become apparent, e.g., when doing envelope-matching calculations. Therefore, a more accurate definition of effective length is needed. In this regard, it should be noted that there is no a priori reason for choosing the pair $\left(l, g_{0}\right)$; for example, the same thin-lens focal length $\left(\left|\kappa_{0 x, y}\right| l\right)^{-1}$ is obtained with an infinite number of combinations of effective lengths greater than $l$, as defined in Eq. (4), and corresponding hardtop values smaller than $g_{0}$. Intuitively, it is clear that the true equivalent hard-edge model must lie between the "standard" model, defined by the pair $\left(l, g_{0}\right)$, and one that extends farther out into the edges and has a smaller hardtop strength.

Reference [6] discusses different ways to model a smooth-profile quadrupole gradient. We choose a procedure, apparently not universally known or practiced, based on replacing the smooth-profile gradient by a number of thin hard-edge "subquadrupoles," and finding, through matrix multiplication, an equivalent hard-edge quadrupole. From the theory, described in detail in Refs. [21,22], effective lengths are found for the focusing and defocusing planes:

$$
l_{f}=-\frac{\theta_{f} \sin \theta_{f}}{F_{21}}, \quad l_{d}=\frac{\theta_{d} \sinh \left(\theta_{d}\right)}{D_{21}},
$$

where $F_{21}, D_{21}$ are matrix elements corresponding to the focusing and defocusing real quadrupole $(2 \times 2)$ matrices, respectively, i.e., the matrices resulting from multiplying out all the submatrices of the model. Further, the quantities $\theta_{f}, \theta_{d}$ are the roots (nearest to zero) of the following transcendental equations:

$$
\cos \theta_{f}+\frac{1}{2} \theta_{f} \sin \theta_{f}-F_{11}+\frac{1}{2} L F_{21}=0,
$$

$\cosh \left(\theta_{d}\right)-\frac{1}{2} \theta_{d} \sinh \left(\theta_{d}\right)-D_{11}+\frac{1}{2} L D_{21}=0$.

$L$, above, is the extent of the gradient profile that is modeled via thin quadrupole "slices." The real quadrupole is then replaced with a hard-edge lens having and effective length $l_{\text {eff }}=\left(l_{f}+l_{d}\right) / 2$, nearly independent of quadrupole strength, and drift elements on either side with a length $\lambda=\left(L-l_{\text {eff }}\right) / 2$.

The effective hardtop strengths $\kappa_{f, d}=\theta_{f, d}^{2} / l_{f, d}$, on the other hand, are almost perfectly linear with the peak strength of the real quadrupole, $\kappa_{q 0}$. Their average value, $\left(\kappa_{f}+\kappa_{d}\right) / 2$, is taken as the effective strength, $\kappa_{q e}$ (the two strengths differ by $<2 \%$ from the average value for the range of operating conditions of the UMER quadrupoles). Figure 3 shows the results of our analysis for the secondgeneration UMER PC quadrupole. We used 23 submatrices each representing $0.5 \mathrm{~cm}$-thin hard-edge quadrupoles. With this choice, $L=11.5 \mathrm{~cm}$, so the gradient profile wings are covered sufficiently since $g(L / 2) / g(0)=0.04 \%$. We find
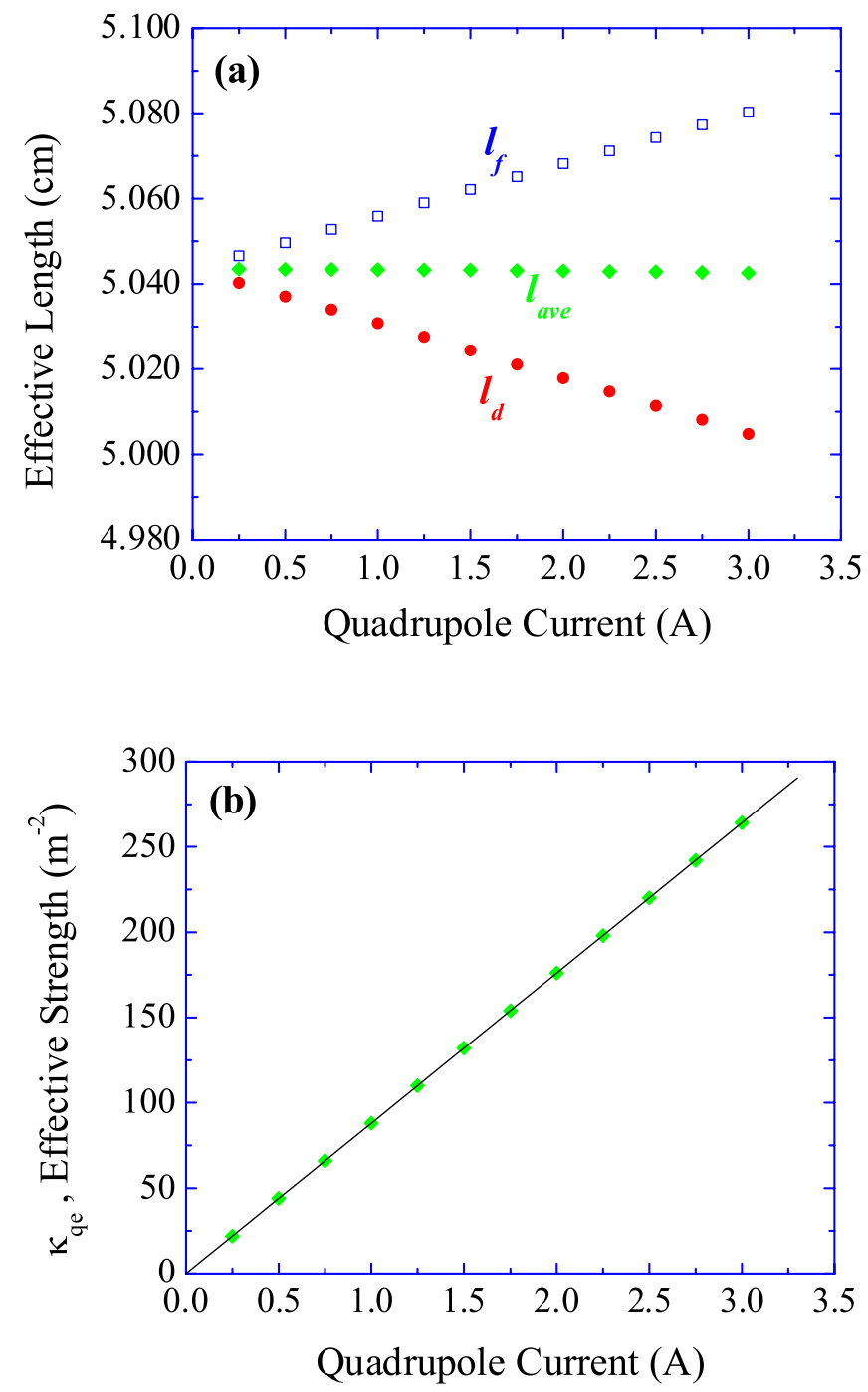

FIG. 3. (Color) Characterization of effective hard-edge model of (2nd generation) UMER magnetic quadrupole: (a) effective lengths in the focusing and defocusing planes and average values as a function of quadrupole current, and (b) effective average strength as a function of quadrupole current [see Eq. (12)].

$$
l_{q e}=5.043 \mathrm{~cm}, \quad \kappa_{q e}\left(m^{-2}\right)=0.72055 \cdot \kappa_{q 0}\left(m^{-2}\right),
$$

the second equality from a linear fit. Figures 2(a) and 2(b) show the two hard-edge models based on Eqs. (4) and (10).

\section{B. Short solenoid}

The on-axis, $z$-component of the $B$-field of the short solenoid employed in the matching section in UMER (Fig. 1) is modeled through the following function developed by one of us (Kishek):

$$
B_{z}(r=o, s)=B_{0} \exp \left(\frac{-s^{2}}{d^{2}}\right)\left[\operatorname{sech}\left(\frac{s}{b}\right)+c_{0} \sinh ^{2}\left(\frac{s}{b}\right)\right],
$$



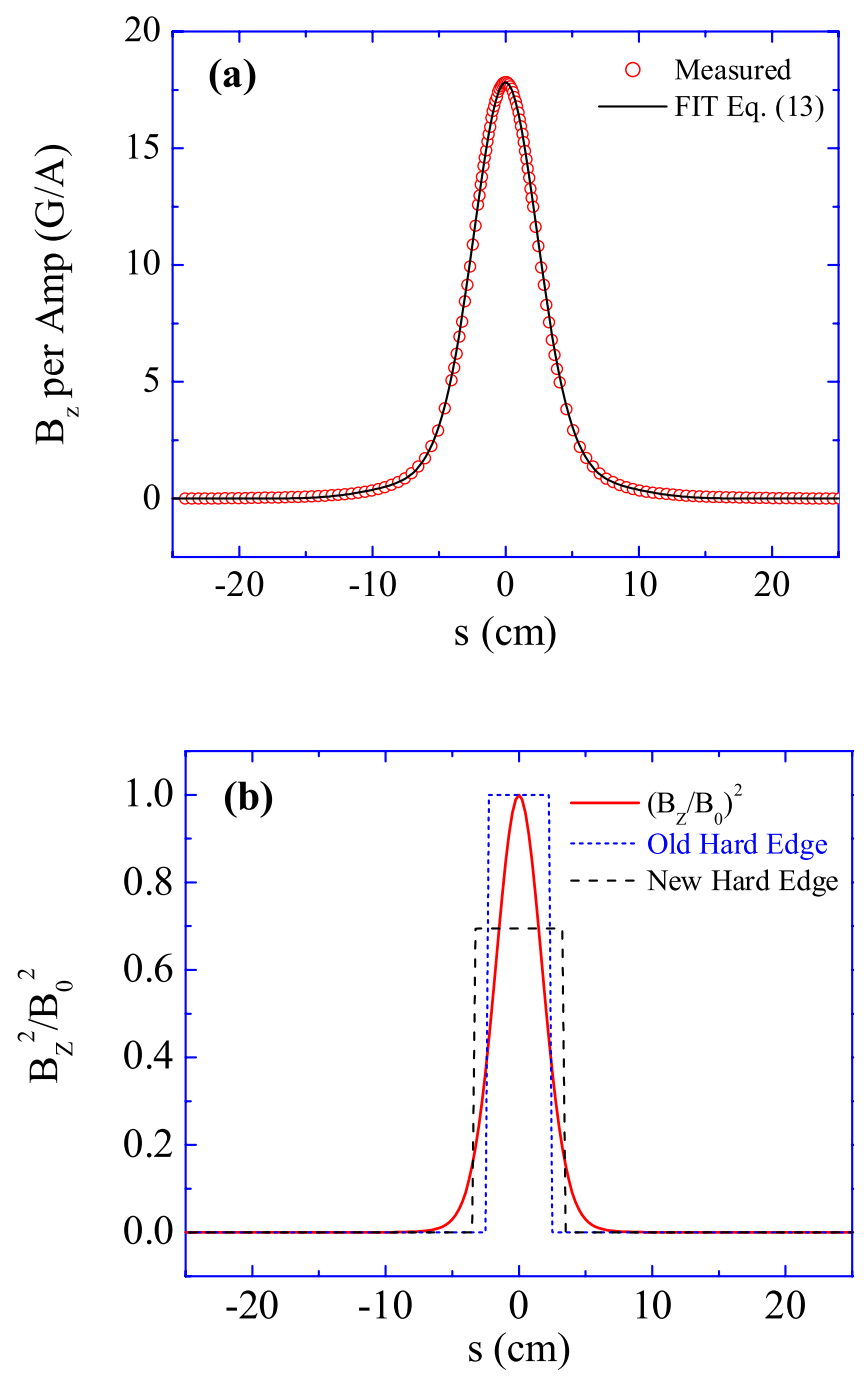

FIG. 4. (Color) Characterization of short solenoid in UMER matching section: (a) measured and analytical fit function [Eq. (13)] of on-axis $B_{z}$ profile, and (b) normalized solenoid strength and hard-edge models.

where $B_{0}$ is the field at the middle plane of the solenoid, and $d=4.81 \mathrm{~cm}, b=3.43 \mathrm{~cm}, c_{0}=0.017$ (dimensionless) are fit constants. Equation (13) is an improvement over the simpler representation [23], $B_{z}(0, s)=$ $B_{0} \exp \left(-s^{2} / d_{o}^{2}\right)\left[1+\left(s^{2} / b_{o}^{2}\right)\right]^{-1} \quad\left(d_{o}=5.65 \mathrm{~cm}, \quad b_{o}=\right.$ $4.16 \mathrm{~cm}$ ). Figure 4(a) illustrates the profile given by Eq. (13) together with $B_{z}(s)$ (measured) per Amp.

By an analysis similar to the one given for the quadrupole [24], we arrive at an equivalent hard-edge model for the solenoid where the effective length is given by

$$
l_{s e}=6.5708 \mathrm{~cm}-0.00029 \cdot \kappa_{s 0}\left(\mathrm{~m}^{-2}\right),
$$

as a function of peak strength, $\kappa_{s 0}$, of the real profile. The corresponding effective hardtop strength is

$$
\kappa_{s e}=0.69448 \cdot \kappa_{s 0}\left(\mathrm{~m}^{-2}\right) .
$$

Figure 4(b) illustrates the standard and new hard-edge models relative to the normalized field profile squared. For reference, the strength profile of the solenoid is given by

$$
\kappa_{s}(s)=\frac{\omega_{L}^{2}(s)}{\beta^{2} c^{2}},
$$

where $\omega_{L}=\left(q / 2 m_{e} \gamma\right) B_{z}(s)$ is the local Larmor frequency, and $B_{z}(s)$ is given by Eq. (13).

Unlike the case with the short quadrupole, care must be taken when computing with the new hard-edge for the solenoid because of the dependence of $l_{s e}$ on the actual peak strength $\kappa_{s 0}$.

\section{PERIODIC FODO MATCHING}

\section{A. Symmetric FODO}

The simplest unit FODO cell consists of two quadrupoles of the same strength but opposite gradient polarities, separated by drifts. The total length of the unit cell is the full-lattice period $S$; the filling ratio is defined as $\eta=$ $l_{q e} /\left[(S / 2)-l_{q e}\right]$, where $l_{q e}=5.043 \mathrm{~cm}$, as calculated above.

We are interested in calculating the quadrupole strengths that are required for a specified zero-current phase advance (per period) $\sigma_{0}$. The simplest calculation employs thin quadrupoles with focal lengths $f= \pm\left(1 / \kappa_{q e} l_{q e}\right)$. We obtain, after straightforward matrix multiplication [25],

$$
\left|\kappa_{q e}\right|=4 \frac{\sin \left(\sigma_{0} / 2\right)}{S l_{q e}} .
$$

The latter equation yields peak quadrupole strengths that are correct within a few percent. However, more accurate and general results can be derived using matrices that include trajectory changes inside the quadrupoles as well as different zero-current phase advances in the two transverse planes. For given zero-current phase advances $\sigma_{0 x}$ and $\sigma_{0 y}$, and filling ratio, the following coupled equations are solved simultaneously for $\theta_{X} \equiv \sqrt{\kappa_{q e x}} l_{q e}$, and $\theta_{Y} \equiv$ $\sqrt{\kappa_{q e y}} l_{q e}$ :

$$
\begin{aligned}
& \cos \left(\sigma_{0 x}\right)=\cosh \left(\theta_{Y}\right)\left[\cos \left(\theta_{X}\right)-\frac{1}{\eta} \theta_{X} \sin \left(\theta_{X}\right)\right]+\sinh \left(\theta_{Y}\right) \sin \left(\theta_{X}\right)\left[\frac{1}{2}\left(\frac{\theta_{Y}}{\theta_{X}}-\frac{\theta_{X}}{\theta_{Y}}\right)-\frac{1}{2 \eta^{2}} \theta_{X} \theta_{Y}+\frac{1}{\eta} \theta_{Y} \cot \left(\theta_{X}\right)\right] \\
& \cos \left(\sigma_{0 y}\right)=\cos \left(\theta_{Y}\right)\left[\cosh \left(\theta_{X}\right)+\frac{1}{\eta} \theta_{X} \sinh \left(\theta_{X}\right)\right]+\sinh \left(\theta_{X}\right) \sin \left(\theta_{Y}\right)\left[\frac{1}{2}\left(\frac{\theta_{X}}{\theta_{Y}}-\frac{\theta_{Y}}{\theta_{X}}\right)-\frac{1}{2 \eta^{2}} \theta_{X} \theta_{Y}-\frac{1}{\eta} \theta_{Y} \operatorname{coth}\left(\theta_{X}\right)\right]
\end{aligned}
$$


These equations are straightforward generalizations of textbook results (see, e.g., [1]). The values of $\kappa_{q e(x, y)}$ yield the required hardtop strengths of the equivalent hard-edge quadrupoles. The actual quadrupole peak strengths $\kappa_{0 x, y}$ are obtained via the second equality in Eq. (12) above, which relates the hardtop and actual peak strengths for the UMER 2nd generation quadrupoles. The corresponding quadrupole currents are then obtained from Eqs. (5) and $g_{0}=4.14 \mathrm{G} / \mathrm{cm} \mathrm{A}$.

As an example from UMER (see Table I for parameters), we find $\kappa_{q e(x, y)}= \pm 165.86 \mathrm{~m}^{-2}$ for $\sigma_{0 x}=\sigma_{0 y}=72.92^{\circ}$. The corresponding peak strength of the actual gradient profile is, from Eq. (12), $\kappa_{0}= \pm 230.2 \mathrm{~m}^{-2}$, or a quadrupole current equal to $1.88 \mathrm{~A}$. We use the envelope code SPOT [26] and the matrix code TRACE 3D [19,20] to find the periodic envelope solution in one period $(S=0.32 \mathrm{~m})$ of UMER. The two codes yield virtually indistinguishable periodic solutions when hard-edge quadrupoles are employed. This is an expected result, since the solution of the $\mathrm{K}-\mathrm{V}$ envelope equations is equivalent to the propagation of the element and beam matrices (which include space charge) used in TRACE [7].

If the quadrupoles are modeled as PMQ, as in the matrix code TRACE [see Eq. (9)], the envelope solutions differ slightly from those in SPOT, which is based on the quadrupole gradient of Eq. (3). The resulting average beam sizes are $1 \%$ larger in TRACE. However, the zero-current phase advance per period, from Eqs. (2), depends slightly on the model: $\sigma_{0}=73.2^{\circ}, 72.1^{\circ}$ in SPOT and TRACE, respectively. Thus, by "retuning" the PMQ strengths in TRACE by $+1 \%$, approximately, to yield the same zero-current phase advance as in SPOT, the differences between the codes all but disappear. Thence, for consistency, we choose to use " $\sigma_{0}$ " in the sense of "zero-current, equivalent hard-edge quadrupole" phase advance per period, with the equivalent hard edge obtained from the Gaussian gradient, Eq. (3) [27].

Figure 5 shows examples from UMER of RMS envelopes in a FODO cell with external focusing given by $\sigma_{0}=$ $72.92^{\circ}$. The $100 \mathrm{~mA}, 10 \mathrm{keV}$ beam is in the extreme spacecharge dominated regime, while the $0.55 \mathrm{~mA}, 10 \mathrm{keV}$ beam is emittance dominated. The quadrupoles were modeled using Eq. (3) in the code SPOT; essentially the same enve-

TABLE I. Beam and lattice parameters in UMER.

\section{Beam current}

Beam energy, $\beta(=v / c)$

Emittance, 4RMS, normalized

FODO period, $S$

Quadrupole $^{\text {a }}$ smooth-profile gradient, $g(s)$

Quadrupole effective length, $l_{q e}$

Solenoid $^{\mathrm{b}}$ smooth-profile, on-axis field, $B_{z}(s)$

Solenoid effective length $l_{s e}-$ see Eq. (14)

${ }^{\mathrm{a}}$ Second generation.

${ }^{\mathrm{b}}$ Matching section only.

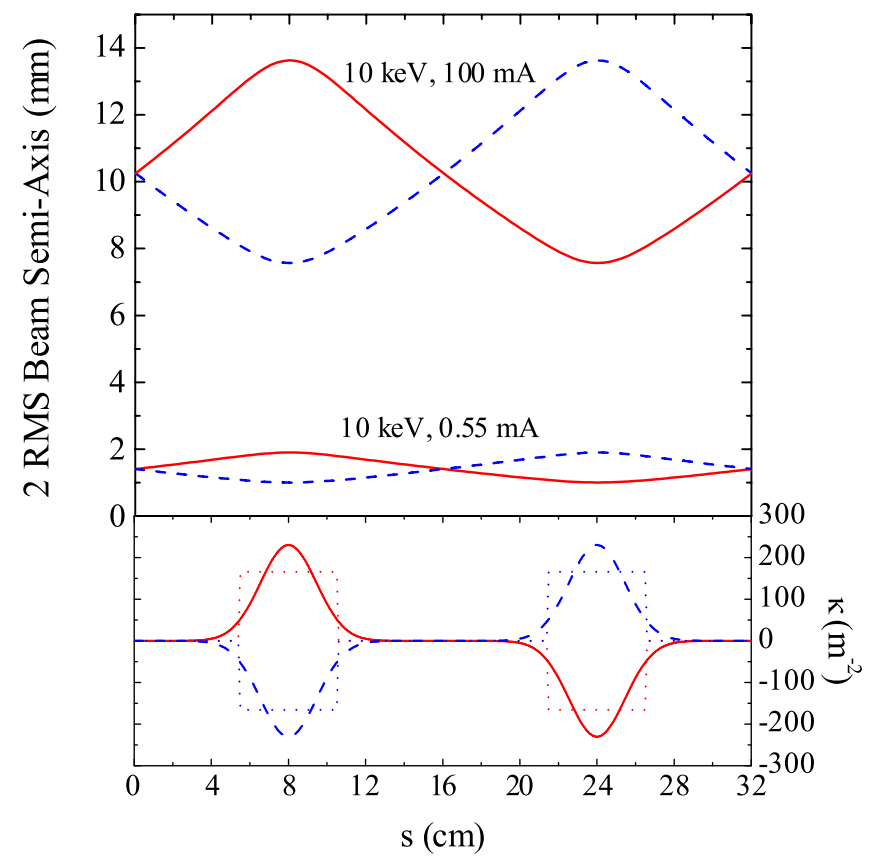

FIG. 5. (Color) Beam envelopes in a unit FODO cell in UMER for $\sigma_{0}=72.92^{\circ}$. Periodic-matched solutions from the envelope code SPOT are shown for extreme space-charge dominated (top) and emittance-dominated (bottom) electron beams (see Table I). The focusing function is proportional to the gradient $g(s)$ of Eq. (3).

lopes are obtained with the refined hard-edge models described above. A characteristic of the periodic envelope solution is its asymmetry about the horizontal line through the crossing point of the $X$ and $Y$ envelopes. Thus, the mean value of $X(z)$ or $Y(z)$ over one period is slightly larger than $X(S / 2)$, or $Y(S / 2)$, i.e., the envelope radius midway between the FODO quadrupoles. The latter quantity is the median of $X(z)$, or $Y(z)$, in one period [28]; its value and the envelope slope $X^{\prime}(S / 2)$, or $Y^{\prime}(S / 2)$, are the relevant quantities that define source-to-FODO matching parameters as discussed in the next section. (Alternatively, one can use $X_{\max }, Y_{\min }$ at the middle plane of the first FODO quadrupole.) We will use in what follows the words "average beam radius" to mean $X(S / 2)$, or $Y(S / 2)$ in a unit FODO cell.

It is interesting to compare the results of numerical RMS envelope matching in a unit FODO cell with simple calculations. The uniform-focusing approximation, as discussed in Chapter 4 of Ref. [1], leads to an expression for the constant beam radius of the matched beam: $a=$ $a_{0}\left(u+\sqrt{1+u^{2}}\right)^{1 / 2}$, where $a_{0}=\left(\epsilon S / \sigma_{0}\right)^{1 / 2}$, and $u \equiv$ $K S / 2 \sigma_{0} \epsilon$. Despite this being a rigorous result, within the approximations discussed in Ref. [1], we prefer to employ another related expression which leads to results closer to the quantity defined above as the average beam radius, a measure obtained from the solution of the K-V envelope equations. Within the uniform-focusing approximation, the 
beam radii in the ideal limits of zero current and zero emittance are given by $a_{0}=\left(\epsilon S / \sigma_{0}\right)^{1 / 2}$, and $a_{B}=$ $K^{1 / 2} S / \sigma_{0}$, respectively. Thus, it is plausible to write an approximate expression for the average beam radius (in the sense explained above) by simply adding $a_{0}$ and $a_{B}$ in quadrature:

$$
a=\frac{S}{\sigma_{0}}\left(\epsilon \frac{\sigma_{0}}{S}+K\right)^{1 / 2},
$$

where $\sigma_{0}=\sigma_{0 x}=\sigma_{0 y}$, and $\epsilon=\epsilon_{x}=\epsilon_{y}$.

For constant zero-current phase advance $\sigma_{0}$, the average beam radius is proportional to $S^{1 / 2}$ in the emittancedominated regime, and to $S$ in the space-charge dominated regime. Thus, a higher density of quadrupoles (larger filling ratio $\eta$ ) facilitates transport of higher currents. Increasing external focusing, which would be the alternative, is not always feasible for practical or for beamdynamics reasons. Power constraints and/or the need to readjust the strengths of a large number of magnets may be impractical, and overfocusing may lead to instabilities and other undesirable effects on the beam. (In UMER, we find it convenient to have to adjust the strengths of only a few magnets in the matching/injection section when changing beam currents.) A larger number of magnets, on the other hand, increases the amplitude of coherent beam oscillations, thus demanding tighter beam/magnet alignment. Fortunately, this increase is slow (as the square root of the number of magnets), and, moreover, is independent (to first order) of RMS-envelope matching.

Table II summarizes results of average beam radii obtained with the codes SPOT and TRACE via smooth-gradient profile models, and calculations using the formula above. (All currents tabulated correspond to UMER experiments.) The use of equivalent hard-edge elements in either code yields essentially the same results as the smooth-profile model in SPOT.

TABLE II. 2RMS average beam radius in a unit FODO cell of UMER for $10 \mathrm{keV}, \sigma_{0}=72.92^{\circ}$. ${ }^{\text {a }}$

\begin{tabular}{ccccc}
\hline \hline $\begin{array}{c}\text { Beam current } \\
(\mathrm{mA})\end{array}$ & $\begin{array}{c}\text { Emittance }^{\mathrm{b}} \\
(\mu \mathrm{m})\end{array}$ & $\begin{array}{c}\text { SPOT }^{\mathrm{c}} \\
(\mathrm{mm})\end{array}$ & $\begin{array}{c}\text { TRACE-3D }^{\mathrm{d}} \\
(\mathrm{mm})\end{array}$ & $\begin{array}{c}\text { Equation (19) } \\
(\mathrm{mm})\end{array}$ \\
\hline 0.55 & 6.0 & 1.4 & 1.4 & 1.4 \\
7.2 & 16 & 3.1 & 3.1 & 3.3 \\
24 & 30 & 5.2 & 5.3 & 5.5 \\
85 & 55 & 9.5 & 9.6 & 9.7 \\
100 & 60 & 10.2 & 10.4 & 10.5 \\
\hline \hline
\end{tabular}

a Zero-current phase advance in hard-edge FODO model, $\left(l_{q e}, \kappa_{q e}\right)=\left(0.05043 \mathrm{~m}, 165.86 \mathrm{~m}^{-2}\right)$.

${ }^{\mathrm{b}}$ 4RMS, unnormalized.

${ }^{\mathrm{c}} \mathrm{K}-\mathrm{V}$ envelope code employing either smooth-profile quadrupoles-Eq. (3), or equivalent hard-edge model.

${ }^{\mathrm{d}}$ Matrix code employing equivalent smooth-profile PMQ quadrupoles -Eq. (9), without "retuning."
TABLE III. Maximum and minimum 2RMS envelope radii in a unit FODO cell of UMER for $10 \mathrm{keV}, \sigma_{0}=72.92^{\circ}$. ${ }^{\mathrm{a}}$

\begin{tabular}{|c|c|c|c|c|c|c|c|}
\hline \multirow{2}{*}{$\begin{array}{l}\begin{array}{l}\text { Beam } \\
\text { current } \\
(\mathrm{mA})\end{array} \\
0.55\end{array}$} & \multirow{2}{*}{$\begin{array}{c}\begin{array}{c}\text { Emittance }^{\mathrm{b}} \\
(\mu \mathrm{m})\end{array} \\
6.0\end{array}$} & \multicolumn{2}{|c|}{$\begin{array}{l}\text { SPOT }^{\mathrm{c}} \\
(\mathrm{mm})\end{array}$} & \multicolumn{2}{|c|}{$\begin{array}{l}\text { Series } \\
\text { expansion }^{\mathrm{d}} \\
(\mathrm{mm})\end{array}$} & \multicolumn{2}{|c|}{$\begin{array}{c}\text { Equation (20) } \\
(\mathrm{mm})\end{array}$} \\
\hline & & 1.9 & 1.0 & 1.9 & 1.0 & 2.1 & 1.0 \\
\hline 7.2 & 16 & 4.1 & 2.3 & 4.3 & 2.4 & 4.8 & 2.4 \\
\hline 24 & 30 & 6.9 & 3.8 & 7.2 & 4.0 & 8.0 & 4.0 \\
\hline 85 & 55 & 12.6 & 7.0 & 12.6 & 7.1 & 14.1 & 7.1 \\
\hline 100 & 60 & 13.6 & 7.6 & 13.6 & 7.6 & 15.2 & 7.7 \\
\hline
\end{tabular}

a Zero-current phase advance in hard-edge FODO model, $\left(l_{q e}, \kappa_{q e}\right)=\left(0.05043 \mathrm{~m}, 165.86 \mathrm{~m}^{-2}\right)$.

b 4 RMS, unnormalized.

${ }^{\mathrm{c}} \mathrm{K}-\mathrm{V}$ envelope code with smooth-profile or equivalent hard-edge quadrupoles.

'Lee's approach [15] and average radii from Eq. (19) or Table II.

The maximum and minimum beam envelope excursions in the periodic lattice are also very important for design as well as beam-dynamics considerations. We can combine results from a simple matrix calculation involving thin lenses over half the lattice period [25], and the approximation $R_{\max , \min }=a\left(\sigma_{0} / S\right)^{1 / 2} \beta_{0 \max , \min }[1,29]$ ( $\beta_{0}$ is the zerocurrent amplitude or betatron function), to obtain

$$
R_{\max , \min }=a\left[\sigma_{0} \frac{1 \pm \sin \left(\sigma_{0} / 2\right)}{\sin \sigma_{0}}\right]^{1 / 2}
$$

where $a$ can be estimated from Eq. (19), and the $+(-)$ sign applies to the maximum (minimum) beam radius. Some improvement over this result is possible if thicklens matrices are employed [in the same spirit as Eqs. (18)]. However, more accurate results can be obtained with Lee's series-expansion approach [15] when applied to the hard-edge FODO model. Table III summarizes results for the same beam parameters of Table II. The calculations with the smooth lattice based on the quadrupole gradient of Eq. (3), on the other hand, are best performed by direct solution of the K-V envelope equations. (The seriesexpansion approach involves, unfortunately for this case, prohibitively involved algebra.) Finally, the ratio $R_{\max } / R_{\min }$ varies from 1.8 (7.2, 24, and $100 \mathrm{~mA}$ beams) to $1.9(0.55 \mathrm{~mA})$, approximately. Thus, the UMER lattice provides slightly smoother focusing for highly spacecharge dominated beams; containment of these beams is made possible with a relatively high density of quadrupoles in the lattice.

\section{B. Asymmetric FODO}

It was assumed in all FODO matching calculations above that both net focusing and emittance were the same in the two transverse planes, i.e., $\sigma_{0 x}=\sigma_{0 y}, \epsilon_{x}=$ $\epsilon_{y}$. Beam transport with asymmetric focusing $\left(\sigma_{0 x} \neq \sigma_{0 y}\right)$ 
and/or asymmetric emittance is also possible in principle. As for symmetric focusing/emittance, a smoothapproximation model can be constructed [30], so effective beam radius $a$, zero-current phase advance, $\sigma_{0}$, and others quantities can be defined. Highly asymmetric beam transport is relevant, for example, to sheet beams of the type employed in klystrons or "flat" beams like the ones envisioned for the International Linear Collider. In this subsection, we briefly consider an example from UMER. We approach the problem by directly solving the envelope equations and comparing the results to approximations similar to those in [30].

We illustrate in Fig. 6 one case of envelope matching in a highly asymmetric FODO cell: $\sigma_{0 x}=57.16^{\circ}, \sigma_{0 y}=$ $73.41^{\circ}$, or $\kappa_{q e 1}=143.20 \mathrm{~m}^{-2}, \kappa_{q e 2}=159.59 \mathrm{~m}^{-2}$, from Eqs. (18). The actual peak strengths are, from Eq. (12), $\kappa_{01}=198.74 \mathrm{~m}^{-2}, \kappa_{02}=221.48 \mathrm{~m}^{-2}$. (The labels " 1 " and " 2 " in the notation refer to the first and second quadrupoles in the asymmetric FODO cell of Fig. 6; obviously, $\kappa_{x}=-\kappa_{y}$ for each quadrupole.) From Fig. 6, we see that the beam is round in two planes around the location of the stronger quadrupole. (If the asymmetry is made even larger, the two transverse envelopes may become "disconnected," so the beam is never round.) Further, the "average" beam radii, $a_{x}, a_{y}$, in the transverse planes can be estimated by solving the algebraic equations

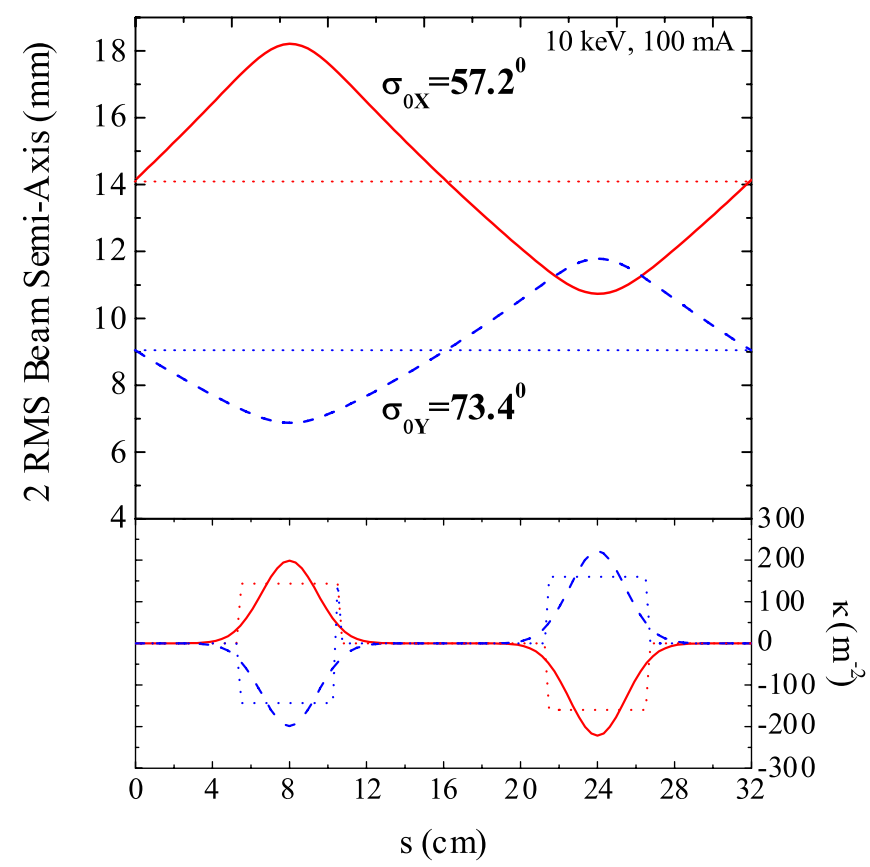

FIG. 6. (Color) Asymmetric FODO matching: beam envelopes in UMER for $\sigma_{0 x}=57.2^{\circ}, \sigma_{0 y}=73.4^{\circ}$. Periodic-matched solutions from the envelope code SPOT are shown for a extreme space-charge dominated beam $(10 \mathrm{keV}, 100 \mathrm{~mA}, 60 \mu \mathrm{m}$-edge emittance).

$$
\begin{aligned}
& \frac{\sigma_{0 x}^{2}}{S^{2}} a_{x}-\frac{2 K}{a_{x}+a_{y}}-\frac{\epsilon_{x}^{2}}{a_{x}^{3}}=0, \\
& \frac{\sigma_{0 y}^{2}}{S^{2}} a_{y}-\frac{2 K}{a_{x}+a_{y}}-\frac{\epsilon_{y}^{2}}{a_{y}^{3}}=0,
\end{aligned}
$$

which are derived along the same lines as for the uniformfocusing approximation with symmetric focusing [1,30,31]. For the example illustrated in Fig. $6(100 \mathrm{~mA}$, $10 \mathrm{keV}, 60 \mu \mathrm{m})$, solution of Eqs. (21) yields $a_{x}=$ $13.8 \mathrm{~mm}, a_{y}=8.6 \mathrm{~mm}$. For comparison, the periodic solution of the K-V envelope equations in SPOT yields $a_{x, y}=$ 14.1, $9.0 \mathrm{~mm}$ with either smooth or equivalent hard-edge quadrupoles. Also from the uniform-focusing approximation, the depressed phase advances per period are obtained from

$$
\begin{aligned}
& \sigma_{x}=\left[\sigma_{0 x}^{2}-\frac{2 K S^{2}}{a_{x}\left(a_{x}+a_{y}\right)}\right]^{1 / 2}, \\
& \sigma_{y}=\left[\sigma_{0 y}^{2}-\frac{2 K S^{2}}{a_{y}\left(a_{x}+a_{y}\right)}\right]^{1 / 2} .
\end{aligned}
$$

The solution of these equations yields tune depressions $\sigma_{x} / \sigma_{0 x}=0.10$, and $\sigma_{y} / \sigma_{0 y}=0.20$. Direct calculation using the $X$ and $Y$ periodic envelopes from SPOT in Eqs. (2) yields $\sigma_{x} / \sigma_{0 x}=0.11$, and $\sigma_{y} / \sigma_{0 y}=0.18$.

Focusing with the large asymmetry illustrated in the example is not normally needed in practice. However, it remains an interesting case to be implemented in UMER for investigations of beam stability and equipartitioning. The actual source-to-FODO matching corresponding to the example just presented is discussed at the end of the next section. A similar implementation of asymmetric focusing in UMER with a $7.2 \mathrm{~mA}, 10 \mathrm{keV}$ beam was reported recently [32].

\section{SOURCE-TO-FODO RMS ENVELOPE MATCHING}

\section{A. Matching problem and optimization}

There are four conditions that the RMS envelopes of a matched beam in a periodic FODO lattice must satisfy: $X(s+S)=X(s), \quad Y(s+S)=Y(s), \quad X^{\prime}(s+S)=X^{\prime}(s)$, and $Y^{\prime}(s+S)=Y^{\prime}(s)$, where $S$ is the lattice period, and prime denotes $d / d s$. Since the beam envelope at the exit plane of the source almost never has the right RMS size and slope for matched transport in a periodic lattice, a matching section is required. Typically, the geometry of matching sections is dictated by physical constraints related to the source, diagnostics, acceleration modules, injection, etc. In all cases, however, a target or terminal beam state for matching can be specified at a convenient plane, e.g., the midplane of the first quadrupole in the periodic lattice, or the plane midway between quadrupoles in the first FODO cell. We employ the latter for the calculations presented 
here. Furthermore, we define the target-envelope parameters from the results employing a smooth-gradient FODO cell based on Eq. (3), as in Fig. 5 (symmetric FODO) or Fig. 6 (asymmetric FODO). Thus, the target parameters (see also Table II) are $\left(X_{f},\left|X_{f}^{\prime}\right|\right)=(10.25 \mathrm{~mm}$, $44.28 \mathrm{mrad}),(1.40 \mathrm{~mm}, 6.69 \mathrm{mrad})$ for the 100 and $0.55 \mathrm{~mA}$ electron beams, respectively. In both cases the energy is $10 \mathrm{keV}$, and $\sigma_{0 x, y}=72.92^{\circ}$.

The problem of finding a matched solution has computational and practical aspects. The first aspect can be studied in the general framework of control theory [33]. It is concerned with finding a solution that minimizes a given condition, typically the difference between the propagated envelope and the target envelope, or a function of this difference. A major feature of the matching solution found in SPOT (see the Appendix), TRACE and other codes is that it corresponds to a local minimum, i.e., it is the best solution in the vicinity of the set of initial values of the matching parameters (typically the strengths of four or more elements). The problem of finding global minima of matching solutions, on the other hand, is a very complex one. It has been addressed for beam transport with negligible space charge [34] and it is under study for intense-beam transport [35].

Although a number of prescriptions exist for estimating the initial values of the matching variables, at least for "zero" current in simple configurations [3] and in special geometries like Collins and low-beta insertions [4,5], it is unavoidable to use some trial and error. However, a useful criterion is that the resulting envelopes in the nonperiodic section should resemble the periodic envelope as much as possible. Naturally, though, with a fixed matching geometry, the criterion can be closely met only for particular beam parameters, as illustrated for a $10 \mathrm{keV}, 7.2 \mathrm{~mA}$ beam in UMER (Fig. 9). The reference trajectory feature in SPOT (see Appendix) also provides guidance when deciding on the initial values of matching variables before final optimization towards a target envelope. Furthermore, the use of natural problem constraints (see below) and merit functionals can be implemented in existing matching codes [36,37] to produce convergence to a matched solution even when the starting guess is relatively far from the final set.

The practical aspects of the matching problem, which are not completely independent of the computational issues, concern the sensitivity of the solutions. Since multiple matching solutions exist, out of the set of possible solutions some must be preferable to others because of better stability under changes of lens strengths, or initial beam parameters, or simply because the solutions provide better treatment of the beam in the sense that a smoother change of the beam envelope from the source to the main FODO lattice is realized.

Finally, matching based on criteria other than the specification of a target state has been suggested. Examples are matching based on a smooth ("adiabatic") variation of the beam envelope or phase advance per period [38], and RMS emittance optimization [39]. These criteria and similar ones may be specially suited for optimization procedures in particular experiments.

\section{B. Calculations with full beam}

With four conditions to be satisfied at the target plane and seven lenses in the UMER matching section (Fig. 1), the matching problem is underconstrained. In other words, we have more "knobs" than we strictly need. We study the matching solutions in UMER by fixing the strengths of the solenoid and first quadrupole, i.e., we parametrize the solutions by specifying the actual peak focusing strengths, $\kappa_{0 S}, \kappa_{01}$, of the solenoid and Q1. We find that a solution can be found with 4 additional quadrupoles for almost any pair $\left(\kappa_{0 S}, \kappa_{01}\right)$. Figure 7 presents three matching solutions for a $100 \mathrm{~mA}, 10 \mathrm{keV}$ beam over a broad range of focusing parameters, all leading to a periodic envelope solution for $\sigma_{0}=72.92^{\circ}$. Table IV summarizes the corresponding element locations and strengths. Many other solutions could be found in between the solutions shown in Fig. 7. A smooth profile was employed for both the solenoid and the quadrupoles, but the same results are obtained with the hard-edge model developed above.

Although no matching solution is preferable to any other in an ideal linear system like the one implicit in the model leading to Fig. 7, practical considerations reduce the space

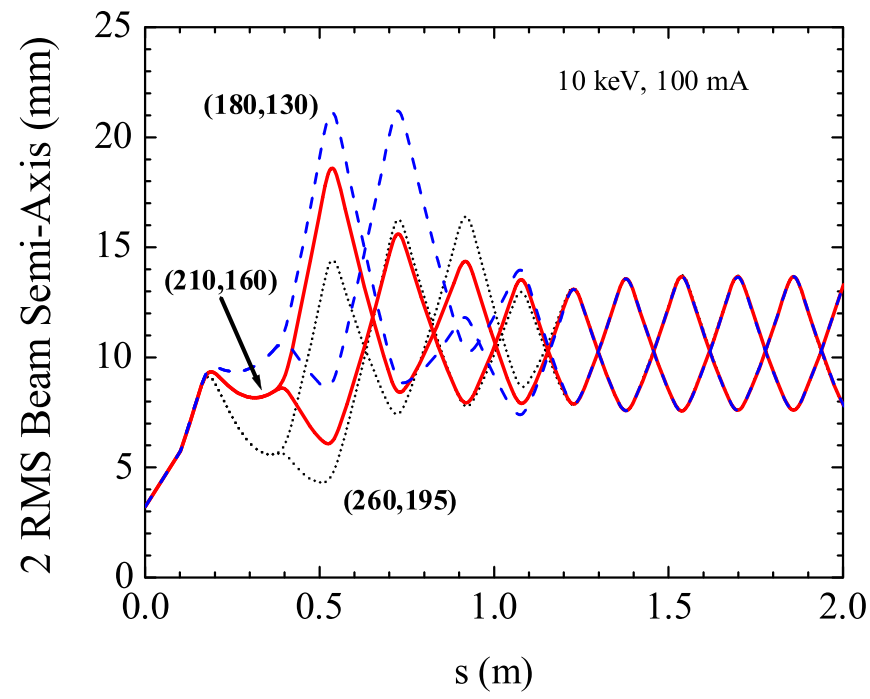

FIG. 7. (Color) Envelope-matching calculations for a $100 \mathrm{~mA}$, $10 \mathrm{keV}, \epsilon=60 \mu \mathrm{m}$ electron beam in UMER. The matching section consists of one solenoid and seven PC quadrupoles (see Fig. 1). Three solutions for $\sigma_{0}=72.92^{\circ}$ are shown (SPOT code with smooth-gradient quadrupoles). The maximum value on the vertical axis corresponds to the vacuum pipe radius. The numbers in parentheses indicate the peak strengths (in $\mathrm{m}^{-2}$ ) of the solenoid and first quadrupole (see Table IV for complete parameters). 
TABLE IV. Parameters for four source-to-FODO matching solutions in UMER with $100 \mathrm{~mA}$, $10 \mathrm{keV}, \epsilon=60 \mu \mathrm{m}$, and $\sigma_{0 x}=\sigma_{0 y}=72.92^{\circ}$. Columns $2-5$ correspond to calculations for the matching/injection section shown in Fig. 1. The last two columns refer to an experiment with a prototype section [40]. See also Figs. 5, 7, and 8.

\begin{tabular}{lccrrrr}
\hline \hline Element & $\begin{array}{c}\text {Distance }^{\mathrm{a}} \\
(\mathrm{cm})\end{array}$ & $\begin{array}{c}(180,130)^{\mathrm{b}} \\
\left(\mathrm{m}^{-2}\right)\end{array}$ & $\begin{array}{c}(210,160) \\
\left(\mathrm{m}^{-2}\right)\end{array}$ & $\begin{array}{c}(260,195) \\
\left(\mathrm{m}^{-2}\right)\end{array}$ & $\begin{array}{r}\text { Distance } \\
(\mathrm{cm})\end{array}$ & $\begin{array}{r}\text { Experiment } \\
\left(\mathrm{m}^{-2}\right)\end{array}$ \\
\hline Solenoid & 17.76 & 180.00 & 210.00 & 260.00 & 16.06 & 241.04 \\
Q1 & 40.04 & 130.00 & 160.00 & 195.00 & 38.89 & 188.70 \\
Q2 & 53.38 & -228.58 & -244.23 & -275.24 & 52.26 & -255.08 \\
Q3 & 72.38 & 204.84 & 225.02 & 246.80 & 71.16 & 233.12 \\
Q4 & 91.94 & -176.12 & -208.30 & -223.51 & 90.80 & -203.99 \\
Q5 & 107.82 & 195.85 & 223.76 & 218.12 & 110.50 & 212.93 \\
Q6 & 122.82 & -235.26 & -228.40 & -216.67 & 133.13 & -208.13 \\
Q7 & 137.82 & 230.20 & 230.20 & 230.20 & 153.57 & 230.20 \\
Q8 & 153.82 & -230.20 & -230.20 & -230.20 & 169.57 & -230.20 \\
\hline \hline
\end{tabular}

${ }^{\mathrm{a}}$ From exit-aperture plane.

${ }^{\mathrm{b}}$ Actual peak focusing strength, $\kappa_{0}$.

of acceptable solutions considerably. The practical constraints in the UMER matching section are common to other machines. The most important constraints are: (i) the maximum beam envelope excursions dictated by the acceptance aperture of focusing elements, (ii) the vacuum pipe radius, (iii) the fixed geometry, and (iv) the power limitations (maximum voltage or current applied to quadrupoles). The first constraint is imposed by the nonlinearities in the magnets and the design "minimum" transport distance, i.e., the desired transport length before unacceptable beam degradation from emittance growth occurs. The second constraint is related to image forces and beam alignment. From Fig. 7, although good beam confinement is accomplished with the solution labeled $(260,195)$, the strong solenoid field of this solution requires tighter beam/ solenoid alignment. Further, most matching quadrupoles under $(260,195)$ (Table IV) are $10 \%-25 \%$ stronger than in the solution labeled $(180,130)$, thus adding to the power requirements. By contrast, $(180,130)$ has large beam excursions (up to 80\% pipe radius, approximately) at Q2 and Q3 and, consequently, a very abrupt transition of the envelope from the matching section to the periodic lattice. Therefore, $(210,160)$, the solid curve in Fig. 7, is a compromise solution that leads to a smoother transition while maintaining modest beam excursions. If the constraint of a fixed geometry is relaxed, on the other hand, it is clear that moving the solenoid together with the rest of the magnets downstream would allow the $100 \mathrm{~mA}, 10 \mathrm{keV}$ beam to expand to reach a radius at Q1 closer to the average radius in the periodic lattice. At the same time, this would yield a smaller beam envelope split at Q2, ie., the difference between $X$ and $Y$ envelope radii. However, a larger beam at the solenoid would also imply a significant increase in effects from the solenoid's spherical aberration. The alternative, which is not feasible in the current UMER matching section, would be to use a quadrupole doublet in place of the solenoid. Even this solution, however, has its problems: (i) very strong magnets are normally required, and (ii) the unavoidable field overlap at the edges is not straightforward to model for accurate matching calculations $[11,17]$.

\section{Experiment with full beam}

A detailed comparison of calculated and observed beam envelopes over the length of the matching section was possible in experiments with a prototype section [11] and with the first realization of the section in UMER [40]. In both experiments, a movable fluorescent screen diagnostic was used to observe the beam from around Q1 out to near Q5. The lattice used was similar to the one shown in Fig. 1, except for a missing quadrupole between Q5 and Q7 and the location of the solenoid (see Table IV). The effect of the additional quadrupole is to eliminate the large beam excursion at the old Q6, while the new location of the solenoid, almost $2 \mathrm{~cm}$ downstream of the previous location, allows the beam to expand more initially, closer to the final average beam radius.

As seen in Fig. 8, there is good agreement between calculated envelope and experiment. Similar agreement was found for other phase advances and beam currents in the space-charge dominated regime [41]. The lattice parameters are summarized in the last two columns of Table IV. Unfortunately, it is not possible to do similar detailed comparisons between experiment and calculation in the current injection section; visual diagnostics are available at only two diagnostic chambers in the straight part of the matching section (IC1 and IC2 in Fig. 1).

\section{Other currents}

The same focusing geometry of Fig. 1 can be used to match all the currents of Table II. Without changing the quadrupole strengths in the periodic FODO lattice, the 


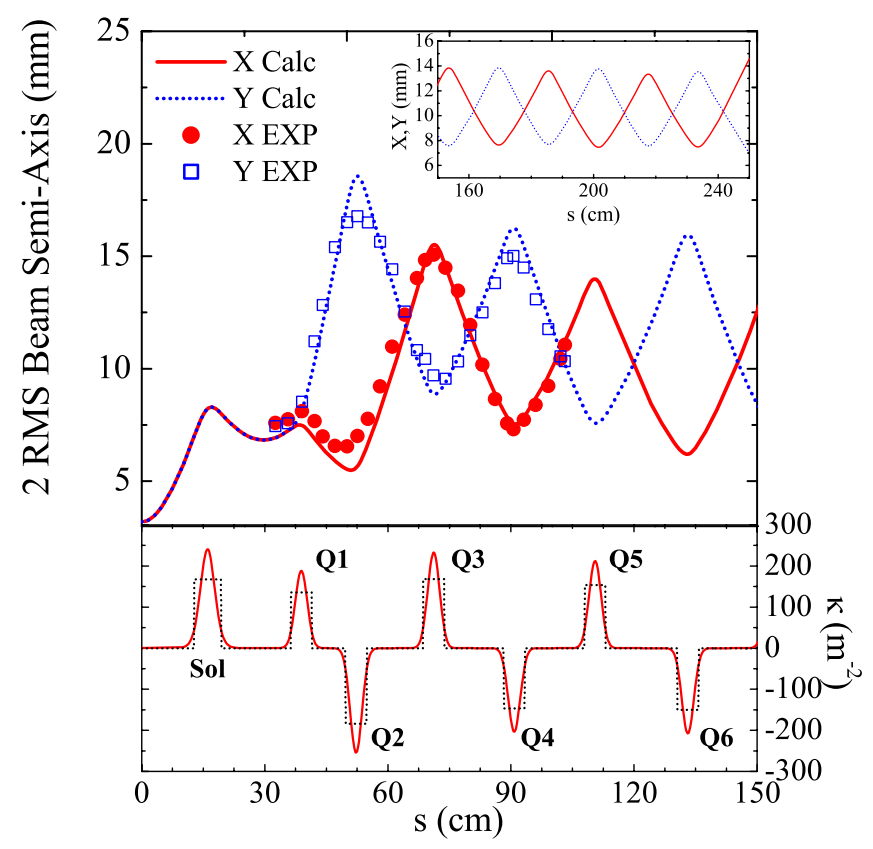

FIG. 8. (Color) Envelope-matching calculations for $\sigma_{0}=$ $72.92^{\circ}$ (from SPOT) vs experimental beam dimensions for a $100 \mathrm{~mA}, 10 \mathrm{keV}, \epsilon=60 \mu \mathrm{m}$ electron beam in UMER. The matching lattice consists of a short solenoid and six PC quadrupoles (see also last two columns in Table IV). The focusing function (one polarity shown) appears at the bottom, including the equivalent hard-edge representation. The inset illustrates the envelope calculated over $1 \mathrm{~m}$ along the periodic lattice, which starts at Q7.

average beam size of a matched beam will vary as $I_{\text {beam }}^{1 / 2}$, approximately, for space-charge dominated beams, or as $\epsilon^{1 / 2}$ for emittance-dominated beams [see Eq. (19)]. Further, as the beam current is reduced, it becomes easier to obtain a smoother transition from the nonperiodic matching section into the periodic FODO lattice, in the space-charge dominated regime. This is so because the solenoid and first quadrupole can be adjusted to split the beam envelope to resemble more the envelope in the periodic lattice. As illustrated in Fig. 9 for the $7.2 \mathrm{~mA}$, $10 \mathrm{keV}$ beam, the location of the beam waist from solenoid focusing is in this case very close to the location of Q1. Thus, comparison with the envelope for the full beam [Fig. 7, solution labeled $(210,160)]$ shows that the envelope radius near Q1 in Fig. 9 is much closer to the average beam radius in the periodic lattice. Further illustration that the matching solution for $7.2 \mathrm{~mA}$ is very well behaved can be seen from the element strengths in Table V, third column: the spread of quadrupole peak focusing strength is small around the periodic lattice value of $230.2 \mathrm{~m}^{-2}$.

In principle, all beams in Table II could be matched with fixed strengths for the solenoid and first two quadrupoles. However, finding matching solutions by varying the required minimum of four elements (for a quadrupole system) normally meets with slow convergence or no con-

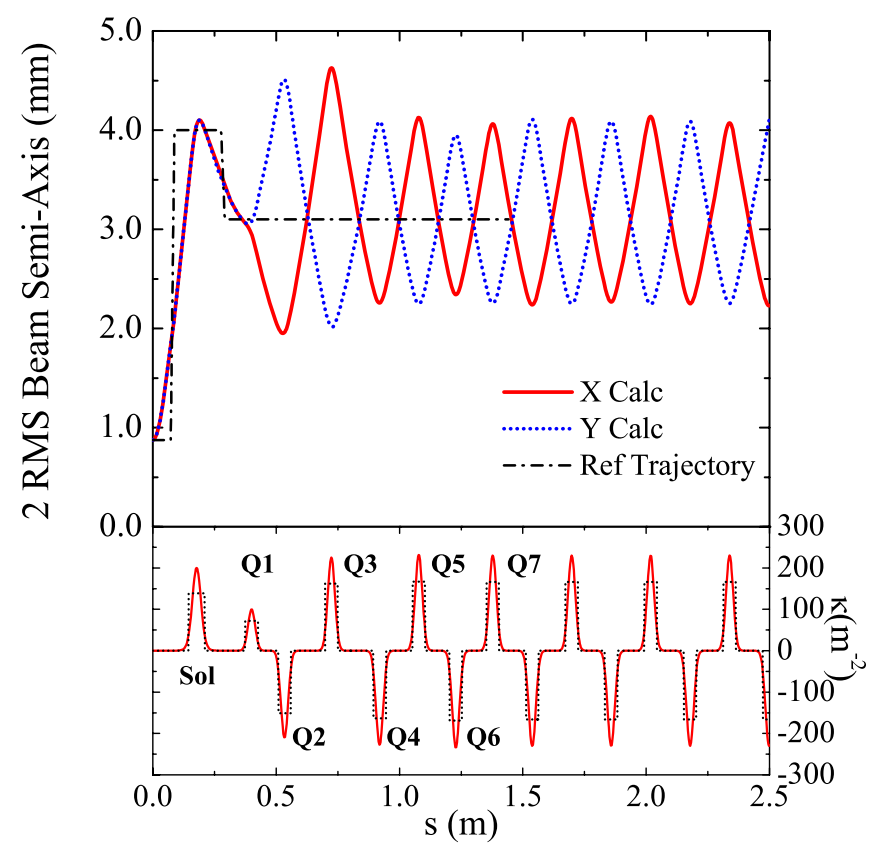

FIG. 9. (Color) UMER RMS-envelope matching with a short solenoid and seven PC quadrupoles (the periodic lattice starts at Q7). Electron beam parameters are: $7.2 \mathrm{~mA}, 10 \mathrm{keV}$, and $\epsilon=$ $16 \mu \mathrm{m}$. Envelope calculations for $\sigma_{0}=72.92^{\circ}$ (from SPOT). The use of the "reference trajectory" for matching is explained in the Appendix. One transverse component of the focusing function is shown at the bottom, including the equivalent hardedge function (see also third column in Table V).

vergence at all, depending on the initial guess for the matching variables and the particular computer code employed. Using too many matching variables, on the other hand, is more computer intensive and can also lead to convergence problems. Thus, we choose to optimize five quadrupoles, Q2-Q6, in most cases, while the solenoid and Q1 can be optimized separately or in a final step. Furthermore, the use of a reference trajectory as an initial step to finding matching solutions in the code SPOT (see the Appendix) works best for relatively well-behaved envelopes. This is the case for space-charge dominated beams in UMER, as shown, e.g., in Fig. 9.

If source-to-FODO matching calculations for the $0.55 \mathrm{~mA}$ beam (emittance dominated) are started using the strengths of all elements that match the beam at $7.2 \mathrm{~mA}$ (Table V), no solution can be found in either SPOT or TRACE. The convergence problem is overcome, though, in a matching code written for MATLAB [42] by one of us (Li); the code takes advantage of a special nonlinear minimization algorithm which is part of MATLAB's optimization toolbox. It is feasible that the same can be accomplished with the standard codes if they are complemented with special optimization routines which involve the implementation of constraints (e.g., a limit on the maximum envelope excursion at a given plane) or merit functionals [36,37]. In any case, keeping the solenoid 
TABLE V. Parameters for (symmetric) source-to-FODO matching solutions in UMER for $7.2 \mathrm{~mA}$ (one solution) and $0.55 \mathrm{~mA}$ (two solutions), $10 \mathrm{keV}, \sigma_{0}=72.92^{\circ}$. The last column corresponds to asymmetric source-to-FODO matching of $100 \mathrm{~mA}, 10 \mathrm{keV}$, with $\sigma_{0 x}=57.2^{\circ}$, $\sigma_{0 y}=73.4^{\circ}$.

\begin{tabular}{lccccc}
\hline \hline Element & Distance & $\begin{array}{c}7.2 \mathrm{~mA}^{\mathrm{b}} \\
\text { Fig. } 9\end{array}$ & $\begin{array}{c}0.55 \mathrm{~mA} \\
\text { Fig. 10(a) }\end{array}$ & $\begin{array}{c}0.55 \mathrm{~mA} \\
\text { Fig. 10(b) }\end{array}$ & $\begin{array}{c}100 \mathrm{~mA} \text {, asymmetric } \\
\text { Fig. 11 }\end{array}$ \\
\hline Solenoid & $17.76 \mathrm{~cm}$ & $200 \mathrm{~m}^{-2}$ & $179.9 \mathrm{~m}^{-2}$ & $168.03 \mathrm{~m}^{-2}$ & $210.0 \mathrm{~m}^{-2}$ \\
Q1 & 40.04 & 100.0 & 110.0 & 0 & 160.0 \\
Q2 & 53.38 & -210.0 & -188.0 & 0 & -254.4 \\
Q3 & 72.38 & 225.0 & 317.0 & 46.71 & 209.3 \\
Q4 & 91.94 & -227.0 & -277.0 & -193.9 & -205.6 \\
Q5 & 107.82 & 231.5 & 284.0 & 248.0 & 217.8 \\
Q6 & 122.82 & -234.7 & -212.0 & -290.9 & -239.7 \\
Q7 & 137.82 & 230.2 & 230.2 & 230.2 & 198.7 \\
Q8 & 153.82 & -230.2 & -230.2 & -230.2 & -221.5 \\
\hline \hline
\end{tabular}

${ }^{\mathrm{a}}$ From exit-aperture plane.

${ }^{\mathrm{b}}$ Actual peak focusing strength, $\kappa_{0}$.

and first quadrupole peak strengths near the values used for $7.2 \mathrm{~mA}$, i.e., 200 and $100 \mathrm{~m}^{-2}$, respectively, yields a solution with large initial envelope variations and overfocusing.

As for the $100 \mathrm{~mA}$ beam, though, the matching solution for $0.55 \mathrm{~mA}$ is hardly unique. Two other solutions for the latter are tabulated in Table V and shown in Figs. 10(a) and 10(b). For the solution labeled "Fig. 10(b)," the first two quadrupoles are powered off, and the third one is fairly weak. One advantage of this solution is the lack of the large envelope split seen at Q2 in solution "Fig. 10(a)." In addition, from the sensitivity study discussed in the next section, "Fig. 10(b)" is the best matching solution of the three calculated for the emittance-dominated beam.

Although all envelope calculations presented here are based on smooth-profile fields and gradients, the results are

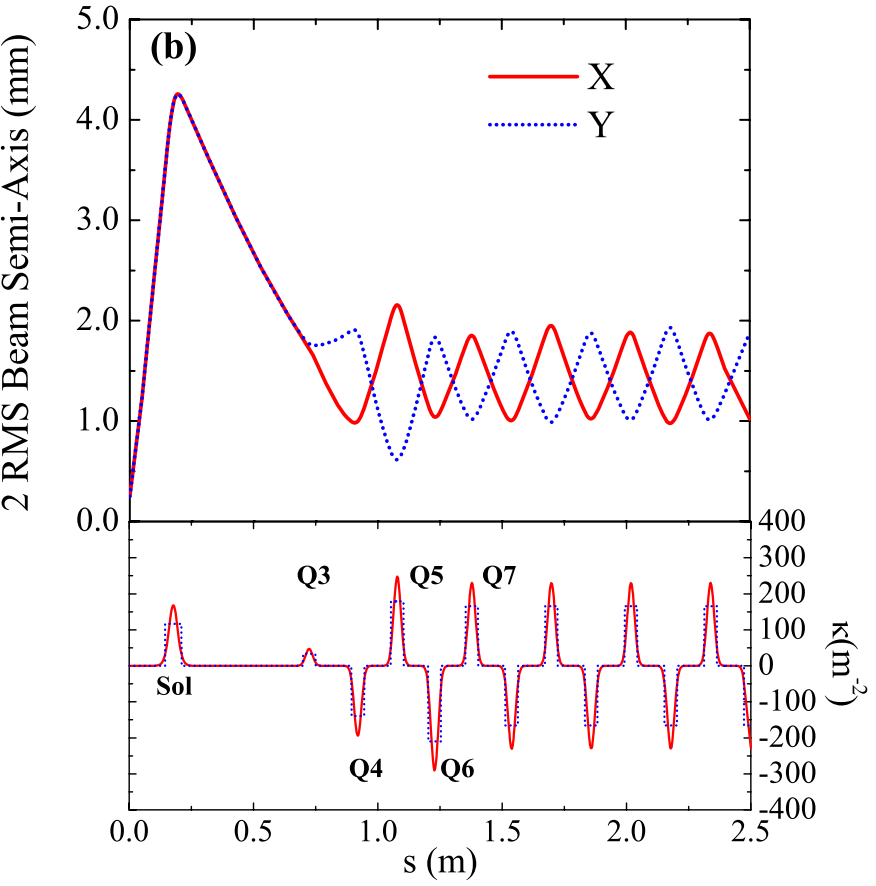

FIG. 10. (Color) Two solutions for UMER RMS-envelope matching with a short solenoid and a number of PC quadrupoles (see also Table V). Electron beam parameters are: $0.55 \mathrm{~mA}, 10 \mathrm{keV}$, and $\epsilon=6.0 \mu \mathrm{m}$. One transverse component of the focusing function is shown at the bottom in each case, including the equivalent hard-edge function. (a) Solution employing all six PC quadrupoles, and (b) solution with four PC quadrupoles (Q1 and Q2 are off). In (a), the envelopes that result from a simple hard-edge model (not the one at the bottom) are also shown. 
indistinguishable from those obtained with the correctly constructed hard-edge focusing functions (Sec. III). Interestingly, the simple (but strictly incorrect) hard-edge model based on Eq. (4) yields sufficiently accurate envelopes, even with focusing elements as short as the UMER solenoid and quadrupoles, in all cases except those where one or both transverse components of the beam envelope undergo large excursions over a relatively short distance. An example is the matching solution in Fig. 10(a) for the $0.55 \mathrm{~mA}$ beam. If the peak strengths from the smooth focusing function are employed in a simple hard-edge model inspired by Eq. (4), the envelopes that result deviate significantly from the correct ones [see Fig. 10(a)]. Thus, properly modeling of the focusing elements is important for all matching calculations. A similar situation has been studied in matching experiments with intense ion beams at Lawrence Berkeley National Laboratory [43]. In the Berkeley experiment, the beam is compressed by a large factor, not unlike the UMER experiment where large compression of one component of the beam envelope is seen at Q2 in Fig. 10(a).

To conclude this section, we present in Fig. 11 the source-to-FODO matching solution corresponding to the example of asymmetric focusing introduced in Sec. IV B above. The geometry of the matching section is the same

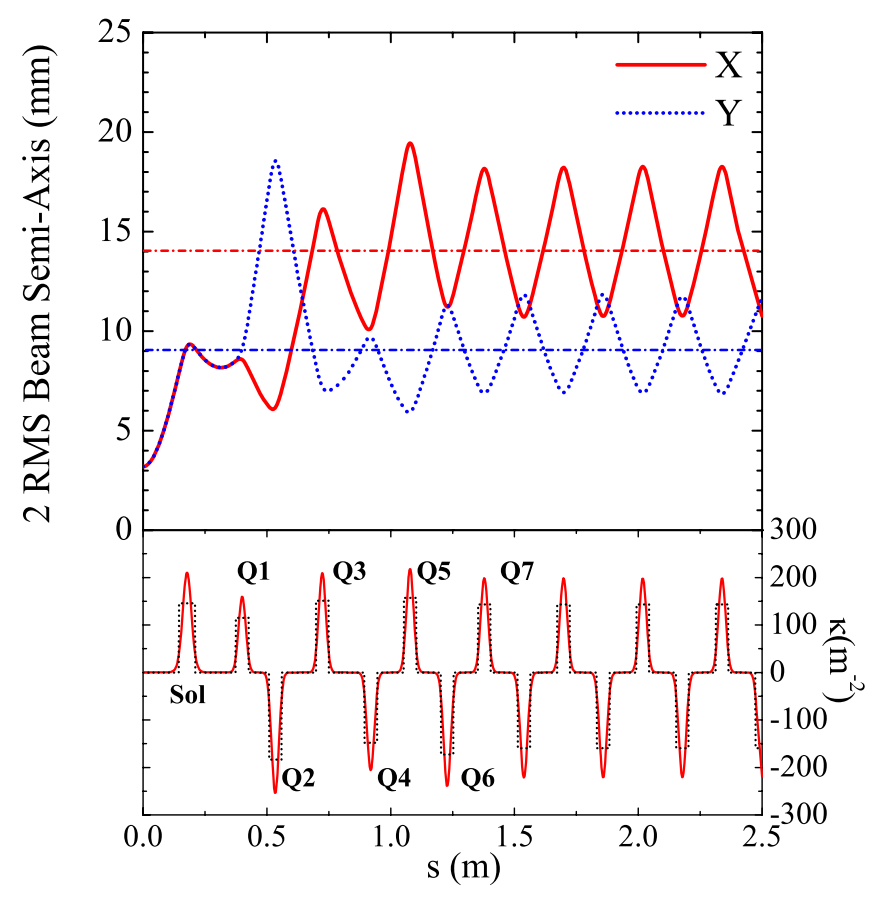

FIG. 11. (Color) UMER RMS-envelope matching with a short solenoid and seven PC quadrupoles. Electron beam parameters are: $100 \mathrm{~mA}, 10 \mathrm{keV}$, and $\epsilon=60 \mu \mathrm{m}$. Calculated beam envelope for $\sigma_{0 x}=57.2^{\circ}, \sigma_{0 y}=73.4^{\circ}$, highly asymmetric (from SPOT). One transverse component of the focusing function is shown at the bottom. The average beam radii indicated by the horizontal broken lines are $a_{x}=14.1 \mathrm{~mm}$, and $a_{y}=9.0 \mathrm{~mm}$ (see also Fig. 6). one used for all tabulated examples; further, the peak strengths of the solenoid and first quadrupole are kept constant at $(210,160) \mathrm{m}^{-2}$ as for the "best" matching solution for symmetric focusing of the $100 \mathrm{~mA}, 10 \mathrm{keV}$ beam (Fig. 7). The target beam parameters, $8 \mathrm{~cm}$ from quadrupole Q8 (i.e., QR1 in Fig. 1), are read off the periodic envelope in Fig. 6: $\left(X_{f}, Y_{f}\right)=(14.45,9.03) \mathrm{mm}$, $\left(X_{f}^{\prime}, Y_{f}^{\prime}\right)=(54.69,-35.87) \mathrm{mrad}$. The strengths of the five quadrupoles that are varied and the two fixed ones that follow are tabulated in the last column of Table V.

\section{SENSITIVITY OF THE MATCHED SOLUTIONS}

The sensitivity of the matched solutions can be studied for changes in element strengths, beam parameters (current, emittance and energy), and initial envelope radius and slope. In addition, quadrupole rotation errors may constitute a source of mismatch. The calculations are complicated by the fact that the target envelope itself, i.e., the envelope in the periodic lattice, is uncertain because of measurement errors of beam parameters. Of these, the emittance has the largest uncertainty, up to $\pm 20 \%$.

An understanding of the sensitivity of the matched solutions under systematic errors in the strength variables can be gained by comparing results from the smoothprofile model and a simple hard-edge model of the lattice focusing function. The latter model is based on the standard definition of effective length [Eq. (4)], which implies a hardtop gradient equal to the peak value of the smooth profile. We find that changes of the order of $3 \%$ in quadrupole strengths have a moderate effect on matching of space-charge dominated beams; this follows from a required retuning of $+3 \%$ of the smooth-profile quadrupoles in a FODO cell to yield the same periodic envelopes as the simple hard-edge model. In UMER, the accuracy and stability of the quadrupole strengths is $<0.3 \%$. The accuracy of the field of the short solenoid at the start of the matching section, on the other hand, is limited by hysteresis and is of the order of $1 \%$.

Monte Carlo calculations were performed to study the effect of random errors. Normal distributions were assumed for the solenoid and quadrupole peak strengths, $\kappa_{0}$, the edge emittances in the two transverse directions and the initial beam envelope parameters $\left(R_{0}\right.$ and $\left.R_{0}^{\prime}\right)$. The 1- $\sigma$ widths of these distributions were assumed, based on measurement errors, to be $2 \%$ for the solenoid peak focusing $\kappa_{0 S}, 1 \%$ for the peak gradient of seven matching quadrupoles, $20 \%$ for each of the transverse edge emittances, and $(0.2 \mathrm{~mm}, 2 \mathrm{mrad})$ for the initial beam envelope parameters $\left(R_{0}, R_{0}^{\prime}\right)$. We obtain 10000 solutions of the envelope equations over the matching section and extract and average the final envelope parameters halfway between Q7 and Q8, i.e., $\left[X\left(s_{f}\right), Y\left(s_{f}\right)\right]$ and $\left[X^{\prime}\left(s_{f}\right), Y^{\prime}\left(s_{f}\right)\right]$ and their standard deviations. We identify the latter as the " $1-\sigma$ matching errors." 
For the $100 \mathrm{~mA}, 10 \mathrm{keV}, 60 \mu \mathrm{m}$ (nominal edge emittance) beam, we find that the main factors contributing to matching errors are the initial beam envelope parameters (at the aperture plate near the electron gun output) and the strengths of the solenoid and Q1. The matching errors from these (combined) factors are $0.5 \mathrm{~mm}$, or $5 \%$ of the nominal matched-envelope dimensions $X_{f}, Y_{f}$, and $4 \mathrm{mrad}$, or about $10 \%$ of the nominal matched-envelope slopes $X_{f}^{\prime}, Y_{f}^{\prime}$. Errors from the remaining variables (the emittances, and strengths of Q2-Q7) are of the same order. Perveance errors, i.e., errors from beam current and/or energy, have a small $(<1 \%)$ effect on the target-envelope parameters. The errors quoted can be translated into mismatch factors (MF) in the sense used in the code TRACE [44]: using the nominal emittance values, the envelope-dimension error alone $(0.7 \mathrm{~mm})$ yields a mismatch factor of order 0.01 , while the net envelope-slope error alone $(6 \mathrm{mrad})$ yields $\mathrm{MF} \simeq 0.5$. The combined errors lead to $\mathrm{MF} \simeq 1$; this degree of mismatch means that mismatch envelope oscillations occur so the envelope occasionally grows by at least a factor of 2 relative to the matched envelope. This prediction is easily verified with additional Monte Carlo calculations over a number, say 10, of lattice periods.

For matching of the emittance-dominated beam $(0.55 \mathrm{~mA}, 10 \mathrm{keV}, 6 \mu \mathrm{m})$, we find, as expected, that errors in the edge emittances are also important factors in addition to errors in the strengths of the solenoid, Q1 and the initial envelope parameters. The uncertainty in the initial beam radius of the emittance-dominated beam is determined by the mechanical tolerance of the aperture employed to obtain it [45]. Monte Carlo calculations around the matching solutions tabulated in Table $\mathrm{V}$ and illustrated in Fig. 10 show that the smallest matching errors are given by the solution labeled "Fig. 10(b)": a net 1- $\sigma$ error of $0.3 \mathrm{~mm}$ for the target envelope dimension, or $20 \%$ of the nominal value, and $3 \mathrm{mrad}$ for the target envelope-slope error, or $45 \%$ of the nominal value. The MF values are 0.08 and 0.9 from envelope dimension and slope errors, respectively; the combined errors yield $\mathrm{MF} \simeq 1$.

Other factors contributing to mismatch that were not considered above include image forces and quadrupole rotation errors. Image forces arise from beam misalignment through the transport line. When the beam centroid is offset by a small amount relative to the vacuum pipe radius, a linear coherent effect results that shifts the zero-current phase advance $\sigma_{0}$ slightly [1]. Larger beam misalignment, though, can further offset the beam and cause distortions and mismatch, especially for space-charge dominated beams. Also, quadrupole rotation errors from assembly and installation produce mismatch, although it is small for the tolerances that are normally achieved in practice.

In UMER, we have conducted transport experiments at $10 \mathrm{keV}$ with $0.55,7.2$, and $24 \mathrm{~mA}$ beams over 24 FODO periods $(2 / 3$ of the ring, or about $9 \mathrm{~m})$. In these experiments, fluorescent screen diagnostics at every other FODO

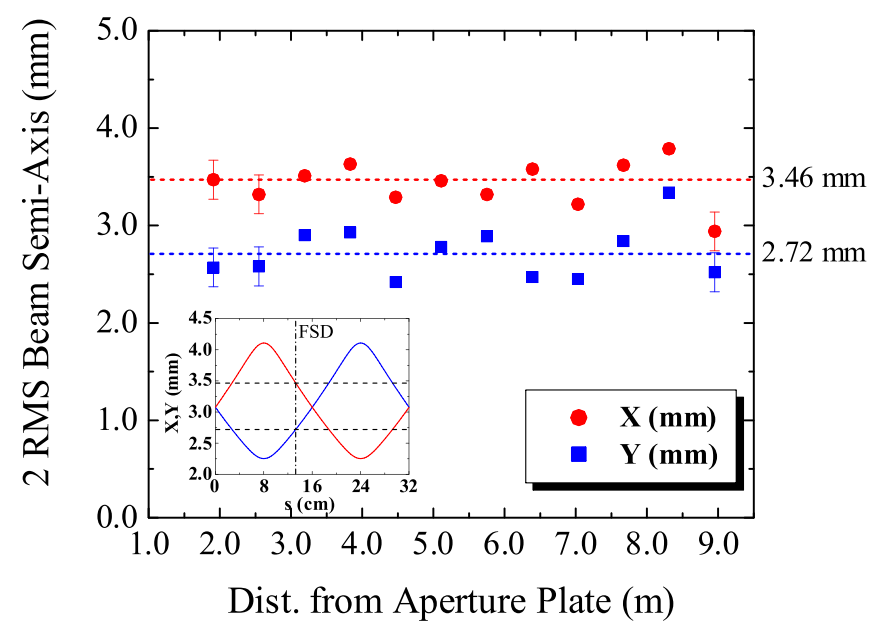

FIG. 12. (Color) Measured beam transverse dimensions (X: horizontal, Y: vertical) over 24 FODO periods in UMER for a $7.2 \mathrm{~mA}, 10 \mathrm{keV}$, electron beam. The horizontal dashed lines represent calculated beam dimensions for $\sigma_{0}=72.92^{\circ}$ at the fluorescent screen diagnostics (FSD). FSD are available at every other FODO cell (ring chambers labeled "RC" in Fig. 1) at the plane indicated by the vertical dotted line in the inset. More details of similar experiments can be found in [46,47].

period allow direct observation and measurement of beam size. (Details of experiments over $1 / 2$ ring appear in Ref. [46].) We have observed envelope oscillations from mismatch in all cases, despite efforts at systematic steering, quadrupole skew correction, and empirical matching. The latter seek to minimize the variance of transverse beam sizes by iteratively varying the strengths of Q1-Q4 using the calculated strengths as the initial values [47]. A moderate reduction in mismatch oscillations is obtained for 7.2 and $24 \mathrm{~mA}$ : from $0.4 \mathrm{~mm}$ to $0.2 \mathrm{~mm}$, approximately, for $24 \mathrm{~mA}$ as reported in [47]. The results of beam size measurements for $7.2 \mathrm{~mA}$ over 24 FODO periods are shown in Fig. 12. Since the fluorescent screen diagnostics are placed at planes $2.7 \pm 0.1 \mathrm{~cm}$ from the midplane in a FODO cell, the expected beam dimensions are different from the tabulated average beam size (Table II). The inset of Fig. 12 illustrates this point.

Current and future experiments in UMER involve pulsed injection for multiturn transport, so direct observation of the beam is only possible on extraction. Emittance measurements at extraction will provide the ultimate test for matching, although efforts will continue for optimizing beam transport over the first turn.

\section{SUMMARY AND CONCLUSIONS}

We have reviewed the basic calculations for RMS envelope matching of beams in magnetic quadrupole systems. We emphasize the importance of properly constructing hard-edge equivalent focusing functions, which is nontrivial for the short focusing elements in UMER. For given lattice and (hard-edge) quadrupole geometries and zero- 
current phase advance per period, formulae are employed to find the required hardtop gradient values of quadrupoles in symmetric or asymmetric FODO cells [Eqs. (18)]. The actual peak strengths (of the smooth-gradient profiles) are then obtained from relations specific to the smooth-profile model [Eq. (12) for UMER].

The average beam radius, as well as the maximum and minimum excursions of the beam envelope in the periodic lattice can be estimated with simple expressions [Eqs. (19) and (20)], or calculated accurately with the seriesexpansion approach of Ref. [15], which is simple enough with hard-edge quadrupoles. Alternatively, K-V envelope or matrix codes employing realistic smooth-profile focusing functions can be used.

Matching of space-charge dominated as well as emittance-dominated beams was shown to be possible by means of the same nonperiodic lattice geometry. The relatively high density of quadrupoles in the UMER lattice makes possible to contain the (extreme) space-charge dominated beams. Further, the question of matching optimization is considered and specifics presented in connection with the envelope code SPOT (Appendix). Although a globally optimized matching solution is desirable in principle, practical considerations reduce the solution space considerably and suggest that a few envelope solutions can be chosen. In this way, well-behaved matching solutions are possible for every beam current despite the limitations of a fixed lattice geometry.

Finally, the sensitivity of the matching solution was studied through Monte Carlo calculations. The results suggest that mismatch oscillations in UMER may, in the worst case (and within a linear model, though), yield to eventual doubling of the maximum beam envelope excursions. Additional effects like image forces and effects from quadrupole rotation errors complicate matters for the transport of space-charge dominated beams. Experimental results of space-charge dominated transport over the length of the matching section $(\sim 1 \mathrm{~m})$ and 24 FODO periods ( $9 \mathrm{~m})$ are also presented. Future work includes refined matching calculations for the new pulsed-injection geometry in UMER, and experiments with asymmetric focusing.

\section{ACKNOWLEDGMENTS}

We thank N. Moody for help with the MATHEMATICA code for Monte Carlo calculations, D. Sutter for reading the manuscript and making useful suggestions, and C. Tobin for pointing out some references. The work is supported by the U.S. Department of Energy.

\section{APPENDIX: THE ENVELOPE CODE SPOT}

We have chosen the envelope code SPOT [26,33] for most matching calculations here because of its sound foundation on control theory, and for its speed and capabilities. As examples of the latter, smooth-profile focusing functions can be defined in SPOT for both solenoids and quadrupoles, a feature not readily available in other codes (e.g., TRACE). In a few special cases where convergence problems arose, though, the calculations were started with MENV, an envelope solver/optimizer written in MATLAB [42] by one of us (Li).

The code SPOT solves the two-dimensional envelope equations, Eqs. (1), for either a unit FODO cell or a general matching section. In the first case, the periodic envelope solution is found for given beam parameters (generalized perveance and edge emittances) and fixed quadrupole strengths corresponding to desired zero-current phase advances. As discussed above, these quadrupole strengths can be found from Eqs. (18) for hard-edge gradient models. The other, more interesting, case of matching is the optimization of a nonperiodic envelope leading from the input plane at $s=s_{i}$ to the periodic envelope starting at $s=s_{f}$. A major feature of SPOT is the use of a reference trajectory. If the average values, in the sense explained in the text, of the beam excursions $X$ and $Y$ are known, the reference trajectory(ies) can be set to be equal to those constant values, except at the beginning of the matching section (see Fig. 9). With a specified reference trajectory and an initial guess for the strengths of the matching lenses, SPOT minimizes the functional given by

$$
J[X(s), Y(s)]=\frac{1}{2} \int_{s_{i}}^{s_{f}}\left\{[X(s)-\bar{X}(s)]^{2}+[Y(s)-\bar{Y}(s)]^{2}\right\},
$$

where $X(s), Y(s)$ are the solutions of Eqs. (1), and $\bar{X}(s)$, $\bar{Y}(s)$ represent the reference trajectory(ies). In essence, $J$ measures the squared distance between the solution envelope and the reference trajectory. A boundary term $\Phi$, which includes the target beam parameters, may be added to $J$. The part of $\Phi$ that specifies the target-envelope transverse dimensions is given by

$$
\Phi\left[X\left(s_{f}\right), Y\left(s_{f}\right)\right]=\frac{1}{2}\left[X\left(s_{f}\right)-X_{f}\right]^{2}+\left[Y\left(s_{f}\right)-Y_{f}\right]^{2},
$$

where $X_{f}, Y_{f}$ are the (2RMS) target-envelope dimensions. Thus, the functional that SPOT minimizes can be chosen to be $J+\Phi$. Normally, the control variables (element peak strengths in most cases) are optimized by minimizing $J$ first, and then adding $\Phi$. In addition, the optimizer can be "tuned" by weighting the terminal state, i.e., by specifying a tuple $\left(W_{1}, W_{2}, W_{3}, W_{4}\right)$ of terminal weights. Thus, the expression in square brackets on the right-hand side in Eq. (A2) would be replaced by

$$
W_{1}\left[X\left(s_{f}\right)-X_{f}\right]^{2}+W_{3}\left[Y\left(s_{f}\right)-Y_{f}\right]^{2},
$$

and similarly for the target-envelope slopes. The path taken during optimization may vary greatly depending on the terminal weights, so increasing their values should nor- 
mally help when the optimizer is not converging to the terminal state.

The optimizing techniques in SPOT utilize tools from nonlinear programming developed for control problems in engineering and mathematics. Additional details can be found in $[26,33]$ and in the documentation to SPOT [48].

[1] M. Reiser, Theory and Design of Charged-Particle Beams (Wiley \& Son, New York, 1994), and references therein.

[2] Ronald C. Davidson and Hong Qin, Physics of Intense Charged Particle Beams in High Energy Accelerators (World Scientific, Singapore, 2001), and references therein.

[3] P. J. Bryant, in Proceedings of CERN Accelerator School: 5th General Accelerator Physics Course, Jyväskylä, Finland, 1992, edited by S. Turner (CERN, Geneva, 1994), Vol. 1, p. 159.

[4] Helmut Wiedemann, Particle Accelerator Physics, Basic Principles and Linear Beam Dynamics (Springer-Verlag, Berlin, 1993), pp. 212-221.

[5] Handbook of Accelerator Physics and Engineering, edited by Alexander $\mathrm{Wu}$ Chao and Maury Tigner (World Scientific, Singapore, 1999), pp. 60-65.

[6] Steven M. Lund and Boris Bukh, Phys. Rev. ST Accel. Beams 7, 024801 (2004).

[7] Christopher K. Allen and Nicholas D. Pattengale, Report No. LA-UR-02-4979, Los Alamos National Laboratory, 2002 (unpublished).

[8] L. Ahle et al., in Proceedings of the 1999 Particle Accelerator Conference, New York, New York (IEEE, New York, 1999), p. 3248.

[9] H. Liu and D, Neuffer, in Proceedings of the 1995 Particle Accelerator Conference, Dallas, Texas (IEEE, New York, 1996), p. 1867.

[10] P. G. O'Shea et al., Nucl. Instrum. Methods Phys. Res., Sect. A 464, 646 (2001).

[11] S. Bernal, P. Chin, R. A. Kishek, Y. Li, M. Reiser, J. G. Wang, T. Godlove, and I. Haber, Phys. Rev. ST Accel. Beams 1, 044202 (1998).

[12] I. M. Kapchinskij and V. V. Vladimirskij, in Proceedings of the international Conference on High Energy Accelerators, Geneva, 1959 (CERN, Geneva, 1959), p. 274.

[13] Frank J. Sacherer, in Proceedings of the 1971 Particle Accelerator Conference [IEEE Trans. Nucl. Sci. 18, 1105 (1971)].

[14] Ronald C. Davidson, Hong Qin, and Paul J. Channel, Phys. Rev. ST Accel. Beams 2, 074401 (1999).

[15] Edward P. Lee, Phys. Plasmas 9, 4301 (2002).

[16] W. W. Zhang et al., Phys. Rev. ST Accel. Beams 3, 122401 (2000).

[17] Santiago Bernal, Ph.D. thesis, Department of Physics, University of Maryland, College Park, 1999.

[18] K. Halbach, Nucl. Instrum. Methods Phys. Res. 187, 109 (1981).

[19] K. R. Crandall and D.P. Rusthoi, TRACE 3-D Documentation, Report No. LA-UR-97-886, Los Alamos National Laboratory, 1997, Appendix E.
[20] Particle Beam Optics Laboratory Ver. 2.1.0 f, Gillespie Associates, Inc., 2002.

[21] Particle Accelerator Physics, Basic Principles and Linear Beam Dynamics, Ref. [4], pp. 129-30.

[22] Klaus G. Steffen, High Energy Beam Optics (John Wiley \& Sons, New York, 1965), pp. 56-58.

[23] Peter F. Loschialpo, Ph.D. thesis, Department of Physics, University of Maryland, College Park, 1984, p. 12.

[24] S. Bernal, UMER Technical Note UMER-060306-SB, 2006 (unpublished).

[25] P. Schmüser, in CERN Accelerator School Proceedings 87-10, edited by S. Turner, Geneva, 1987.

[26] C. K. Allen, S. K. Guharay, and M. Reiser, in Ref. [9], p. 2324.

[27] Alternatively, if we insist that the two smooth-gradient models, Eqs. (3) and (9), have the same peak strengths and yield the same $\sigma_{0}$, slightly different effective lengths are required.

[28] The difference between mean and median envelope radii is of the order of $1 \%$ in our case $\left(\sigma_{0}=72.92^{\circ}\right)$, and fairly insensitive to filling ratio $\eta$.

[29] The approximation in [1] states that the "ripple function,", i.e., the deviation of the envelope from its average value $a$, is constant and equal to the zero-current value.

[30] Martin Reiser and Hui Li, J. Appl. Phys. 96, 784 (2004).

[31] A major assumption behind this result is that the "ripple function" [29] is the same for both transverse envelopes.

[32] S. Bernal et al., in Proceedings of the 2005 Particle Accelerator Conference, Knoxville, TN, edited by $\mathrm{C}$. Horak (IEEE, New York, 2005), p. 892.

[33] Chris Allen, Ph.D. Thesis, Department of Electrical Engineering, University of Maryland, College Park, 1996.

[34] Yu-Chiu Chao, in Proceedings of the 2001 Particle Accelerator Conference, Chicago, IL (IEEE, New York, 2001), p. 1773.

[35] Eugenio Schuster, Christopher K. Allen, and Miroslav Krstic, in Proceedings of the 2005 Particle Accelerator Conference, Knoxville, TN (Ref. [32]), p. 4269.

[36] G.H. Gillespie, B.W. Hill, J.M. Moore, and M.D. Woodley, in Proceedings of the XX International Linac Conference, Monterrey, California, 2000, p. 306.

[37] G.H. Gillespie, B.W. Hill and J.M. Moore, in Proceedings of the 2000 European Particle Accelerator Conference, Vienna, Austria, 2000, p. 1375.

[38] G. Bellomo, M. Novati, and P. Pierini, in Proceedings of EPAC 2002, Paris, France, p. 1118.

[39] D. Jeon et al., SNS/AP Technical Note Number 09, Spallation Neutron Source Project, Oak Ridge National Laboratory, 2001.

[40] S. Bernal et al., in Proceedings of the 2001 Particle Accelerator Conference, Chicago, IL (Ref. [34]), p. 2129.

[41] S. Bernal et al., Nucl. Instrum. Methods Phys. Res., Sect. A 519, 380 (2004).

[42] MATLAB Version 6.5.0, The MathWorks, Inc., 2002.

[43] L. R. Prost et al., Phys. Rev. ST Accel. Beams 8, 020101 (2005).

[44] See Ref. [19], Appendix C. The rationale for a similar definition of MF is found in C. J. Guyard and M. Weiss, in Proceedings of the 1976 International Linac Conference, Atomic Energy of Canada, AECL-5677 (1976), p. 254.

[45] Naturally, $R_{0}$, the initial beam envelope radius, is equal to 
the radius of the collimating aperture. Only a very exotic beam particle distribution behind the aperture could yield an effective beam size different from the aperture size. The aperture radius is $0.25 \pm 0.02 \mathrm{~mm}$, and the initial envelope slope downstream of the aperture, $R_{0}^{\prime}$, is taken as $0.0 \pm 6 \mathrm{mrad}$. The slope uncertainty corresponds to the maximum envelope slope of the full beam before collimation.
[46] S. Bernal et al., Phys. Plasmas 11, 2907 (2004).

[47] Hui Li et al., in Proceedings of the 15th International Symposium on Heavy Ion Inertial Fusion [Nucl. Instrum. Methods Phys. Res., Sect. A 544, 367 (2005)].

[48] SPOT contains a detailed hyperlinked manual. The code and all files related to this paper are freely available upon request. Please write to sabern@umd.edu. 